\title{
Polygonal Web Representation for Higher Order Correlation Functions of Consistent Polygonal Markov Fields in the Plane
}

\author{
Tomasz Schreiber
}

Received: 1 May 2009 / Accepted: 23 June 2010 / Published online: 13 July 2010

(C) The Author(s) 2010. This article is published with open access at Springerlink.com

\begin{abstract}
We consider polygonal Markov fields originally introduced by Arak in 4th USSR-Japan Symposium on Probability Theory and Mathematical Statistics, Abstracts of Communications, 1982; Arak and Surgailis in Probab. Theory Relat. Fields 80:543-579, 1989. Our attention is focused on fields with nodes of order two, which can be regarded as continuum ensembles of non-intersecting contours in the plane, sharing a number of salient features with the two-dimensional Ising model. The purpose of this paper is to establish an explicit stochastic representation for the higher-order correlation functions of polygonal Markov fields in their consistency regime. The representation is given in terms of the socalled crop functionals (defined by a Möbius-type formula) of polygonal webs which arise in a graphical construction dual to that giving rise to polygonal fields. The proof of our representation formula goes by constructing a martingale interpolation between the correlation functions of polygonal fields and crop functionals of polygonal webs.
\end{abstract}

Keywords Arak-Surgailis polygonal Markov fields · Higher order correlation functions · Polygonal web · Duality between polygonal fields and polygonal webs · Graphical construction · Two-dimensional Ising model

\section{Introduction}

Polygonal Markov fields, originally introduced by Arak and Surgailis [1, 2] and further studied by Arak, Clifford and Surgailis [3, 4, 18] are random ensembles of non-intersecting polygonal contours in the plane interacting by hard-core exclusions and exhibiting twodimensional germ-Markov property [2], with a variety of additional possible terms entering the Hamiltonian, including length and area elements (ibidem). The polygonal fields with

Research supported by the Polish Minister of Science and Higher Education grant N N201 385234 (2008-2010) and from grant of Nicolaus Copernicus University, Toruń, Poland.

T. Schreiber $(\bowtie)$

Faculty of Mathematics \& Computer Science, Nicolaus Copernicus University, ul. Chopina 12/18,

87-100 Toruń, Poland

e-mail: tomeks@mat.uni.torun.pl 
Fig. 1 A typical realisation of a polygonal Markov field with V-shaped nodes

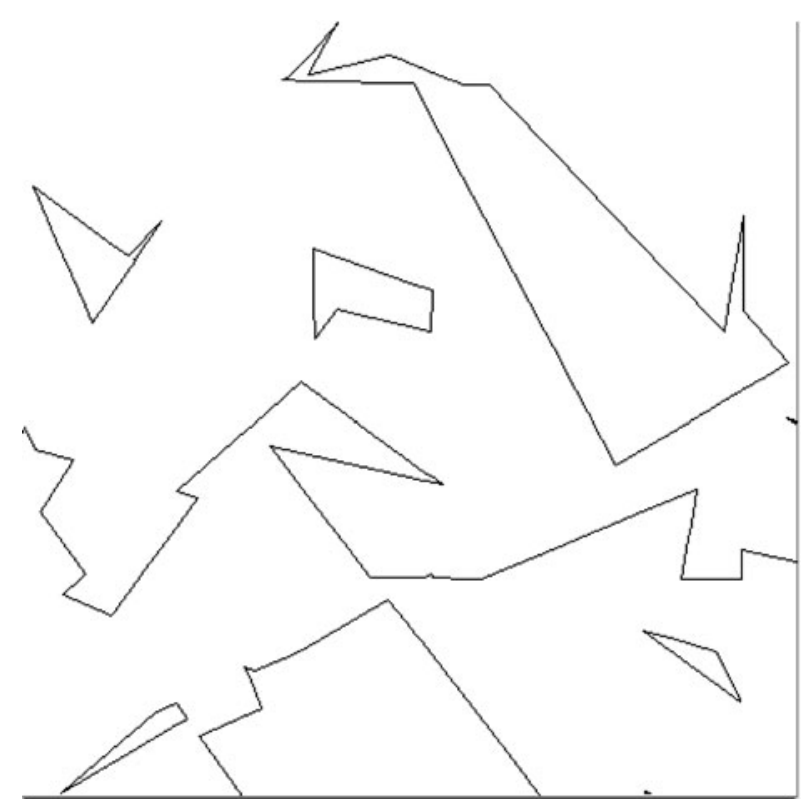

V-shaped nodes (no nodes of order higher than two) as considered in this paper, see Fig. 1 for a typical realisation, share a number of essential features with the two-dimensional Ising model, prominent examples including the presence of an Ising-like phase transition [13, 14] as well as low temperature phase separation and Wulff droplet creation [15]. For these reasons, the polygonal Markov fields are sometimes regarded as continuum counterparts of the Ising model in the plane (as well as of the Potts model if higher order nodes are admitted). Remarkably, in many aspects the polygonal fields are exactly tractable, especially in the so-called consistent regime falling into the supercritical temperature region. In particular, at the consistency point one knows the exact value of the partition function, first and second order characteristics of the field [2-4]. Further, a lot is known about the higher order correlations as well, including certain exact formulae [16] and necessary and sufficient conditions for factorisation of edge correlations (ibidem) as well as an exponential mixing statement (asymptotic factorisation) for edge correlations (ibidem). A striking feature of polygonal Markov fields is that they admit a number of particularly convenient algorithmic constructions-graphical representations [2-4, 10, 14-16] which are in fact the main tool for establishing of the afore-mentioned results. The geometric ingredient in these considerations is so predominant that often no supplementary calculations are needed, which stands in a strong contrast to the classical Ising model.

The rich class of graphical constructions developed for polygonal fields have also found their applications in Bayesian image processing where they are used to generate image segmentations, see $[6,8-10,17]$. Experimenting with various black-white and grayscale images we already obtained promising results, further algorithms are a subject of our ongoing research in progress.

The purpose of the present paper is to complement the existing exact results for consistent polygonal Markov fields by establishing in Theorem 2 an explicit stochastic representation for their higher order edge correlations in terms of expectations of the so-called crop functionals of polygonal webs. The polygonal web arises as the union of a collection of trajectories of continuous time critical branching polygonal random walks in the plane, 
interacting by a bridge-creating mechanism attempting to clasp random trees generated by branching walks into a web by establishing linear bridges between trees. As mentioned above, the polygonal fields admit a graphical construction whose full details are given in Sect. 3 below. The dynamics of this construction can be interpreted as a (quite untypical) interacting particle system evolving in time and, speaking in this vein, the polygonal web arises in a graphical construction, given in Sect. 4 below, which can be regarded as dual to that for polygonal fields. The nature of this duality consists, roughly speaking, in the fact that the dynamic representation of polygonal webs exhibits features strongly reminiscent of those of the polygonal field construction under inverted time flow direction. To some extent this can be perceived as an analogy to the classical duality for interacting particle systems, see e.g. Sect. III.4 in [11], although this is not a close analogy, especially that here we deal with entire histories of the considered interacting particle systems (whose trajectories trace the polygonal fields and webs) rather than just with their instantaneous configurations. The proof of our duality representation for edge correlations goes by constructing a martingale interpolating between edge correlation functions and crop functionals of the corresponding polygonal webs. More precisely, we prove in Sect. 6 that there exists a real-valued edge correlation process $\left(\Phi_{s}\right)_{s \in[0,1]}$ enjoying the following properties

- $\Phi_{0}$ coincides a.s. with the suitably defined edge correlation function $\phi(\cdot)$ as discussed in detail in Sect. 5.

- $\Phi_{1}$ coincides a.s. with the crop functional of the corresponding polygonal web, $\operatorname{crop}(\mathcal{W}[\cdot])$, as defined in detail in Sect. 4.

- $\left(\Phi_{s}\right)_{s \in[0,1]}$ is a martingale as shown in the crucial Lemma 4, which from the technical viewpoint can be regarded as the principal result of this paper.

Putting these together allows us to establish our main Theorem 2 stating that $\phi(\cdot)=$ $\mathbb{E} \operatorname{crop}(\mathcal{W}[\cdot])$, that is to say that the edge correlation function coincides with the expectation of the crop functional of the corresponding polygonal web.

Apart from their intrinsic theoretical interest our results have one further important aim. Namely they provide effective means to evaluate correlation functions and conditional correlations of polygonal fields. In the continuum set-up this can be done using Monte-Carlo techniques based on Theorem 2. However, in our recent work [17] we have developed a hybrid semi-discrete version of polygonal field theory strongly oriented for Bayesian image analysis. We intend to specialise the duality formula in Theorem 2 to that set-up and to obtain in this way an efficient computer algorithm for exact calculation of the field correlations and conditional correlations, without resorting to approximate Monte-Carlo techniques. As a result we will construct an exact Bayesian inference engine on the space of polygonal images. Whereas the theory of Bayesian exact inference engines for belief networks has become very well developed by now, see e.g. [7] and the references therein, these are usually designed for generic nets and do not exploit the particular geometric features as present in our polygonal setting. Further details of this work in progress fall beyond the scope of this article and will be published in a separate paper.

The rest of this paper is organised as follows. In Sect. 2 below we present the concept of a non-homogeneous polygonal field as introduced in [16]. Next, in Sect. 3 we discuss the generalised graphical construction of such fields as developed ibidem. In the further Sect. 4 we develop a dual graphical construction and define the polygonal web. In the next Sect. 5 we define edge correlation functions of polygonal fields and state our main representation Theorem 2 as briefly discussed above. The proof of this theorem is given in Sect. 6, where a number of auxiliary constructions are also developed and many auxiliary results established. Finally, to the last Sect. 7 we delegate the proof of a technical existence result for edge correlations. 


\section{Non-homogeneous Polygonal Markov Fields in the Plane and Their Consistent Regime}

For an open bounded convex set $D$ define the family $\Gamma_{D}$ of admissible polygonal configurations on $D$ by taking all the finite planar graphs $\gamma$ in $D \cup \partial D$, with straight-line segments as edges, such that

- the edges of $\gamma$ do not intersect,

- all the interior vertices of $\gamma$ (lying in $D$ ) are of degree 2,

- all the boundary vertices of $\gamma$ (lying in $\partial D$ ) are of degree 1 ,

- no two edges of $\gamma$ are colinear.

In other words, $\gamma$ consists of a finite number of disjoint polygons, possibly nested and chopped off by the boundary. Further, for a finite collection $(l)=\left(l_{i}\right)_{i=1}^{n}$ of straight lines intersecting $D$ we write $\Gamma_{D}(l)$ to denote the family of admissible configurations $\gamma$ with the additional properties that $\gamma \subseteq \bigcup_{i=1}^{n} l_{i}$ and $\gamma \cap l_{i}$ is a single interval of a strictly positive length for each $l_{i}, i=1, \ldots, n$, possibly with some isolated points added.

For a Borel subset of $A \subseteq \mathbb{R}^{2}$ by [[A]] we shall denote the family of all straight lines hitting $A$ so that in particular $\left[\left[\mathbb{R}^{2}\right]\right]$ stands for the collection of all straight lines in $\mathbb{R}^{2}$. We shall also write $\overline{[}[A] \overline{]}$ for the family of all linear segments in $\mathbb{R}^{2}$ hitting $A$. Further, let $\mu$ be the standard isometry-invariant Haar-Lebesgue measure on the space $\left[\left[\mathbb{R}^{2}\right]\right]$ of straight lines in $\mathbb{R}^{2}$. Recall that one possible construction of $\mu$ goes by identifying a straight line $l$ with the pair $(\phi, \rho) \in[0, \pi) \times \mathbb{R}$, where $(\rho \sin (\phi), \rho \cos (\phi))$ is the vector orthogonal to $l$, and joining it to the origin, and then by endowing the parameter space $[0, \pi) \times \mathbb{R}$ with the usual Lebesgue measure. Note that the above parametrisation of $\left[\left[\mathbb{R}^{2}\right]\right]$ with $[0, \pi) \times \mathbb{R}$ endows $\left[\left[\mathbb{R}^{2}\right]\right]$ with a natural metric, topology and Borel $\sigma$-field which will be used in this paper. Next, consider a non-negative Borel measure $\mathcal{M}$ on $\left[\left[\mathbb{R}^{2}\right]\right]$ admitting a locally bounded density $m(\cdot)$ with respect to $\mu$. Below, the measure $\mathcal{M}$ will be interpreted as the activity measure on $\left[\left[\mathbb{R}^{2}\right]\right]$. Let $\Lambda^{\mathcal{M}}$ be the Poisson line process on $\left[\left[\mathbb{R}^{2}\right]\right]$ with intensity measure $\mathcal{M}$ and write $\Lambda_{D}^{\mathcal{M}}$ for its restriction to the domain $D$. Further, define the Hamiltonian $L^{\mathcal{M}}$ : $\Gamma_{D} \rightarrow \mathbb{R}_{+}$given by

$$
L^{\mathcal{M}}(\gamma):=\sum_{e \in \operatorname{Edges}(\gamma)} \mathcal{M}([[e]]), \quad \gamma \in \Gamma_{D} .
$$

We note that the energy function $L^{\mathcal{M}}$ should be regarded as an anisotropic environmentspecific version of the length functional. Indeed, interpreting the activity $\mathcal{M}(d l)$ of a line $l$ hitting an edge $e \in \operatorname{Edges}(\gamma)$ as the likelihood of a new edge being created along $l$ intersecting and hence fracturing the edge $e$ in $\gamma$, we observe that, roughly speaking, the value of $\mathcal{M}([[e]])$ determines how likely the edge $e$ is to be fractured by another edge present in the environment. In other words, $L^{\mathcal{M}}(\gamma)$ determines how difficult it is to maintain the whole graph $\gamma \in \Gamma_{D}$ without fractures in the environment $\mathcal{M}$-note that due to the anisotropy of the environment there may be graphs of a higher (lower) total edge length than $\gamma$ and yet of lower (higher) energy and thus easier (more difficult) to maintain and to keep unfractured due to the lack (presence) of high activity lines likely to fracture their edges.

Following [16], with the above notation, for $\beta \in \mathbb{R}$ further referred to as the inverse temperature (from mathematical viewpoint also the unphysical negative values of inverse temperatures are admissible), we define the polygonal field $\mathcal{A}_{D}^{\mathcal{M} ; \beta}$ in $D$ with activity measure $\mathcal{M}$ to be the Gibbsian modification of the process induced on $\Gamma_{D}$ by $\Lambda_{D}^{\mathcal{M}}$, with the 
Hamiltonian $L^{\mathcal{M}}$ at inverse temperature $\beta$, that is to say

$$
\mathbb{P}\left(\mathcal{A}_{D}^{\mathcal{M} ; \beta} \in G\right):=\frac{\mathbb{E} \sum_{\gamma \in \Gamma_{D}\left(\Lambda_{D}^{\mathcal{M}}\right) \cap G} \exp \left(-\beta L^{\mathcal{M}}(\gamma)\right)}{\mathbb{E} \sum_{\gamma \in \Gamma_{D}\left(\Lambda_{D}^{\mathcal{M}}\right)} \exp \left(-\beta L^{\mathcal{M}}(\gamma)\right)}
$$

for all sets $G \subseteq \Gamma_{D}$ Borel measurable with respect to, say, the usual Hausdorff distance topology. Note that this definition can be rewritten as

$$
\mathbb{P}\left(\mathcal{A}_{D}^{\mathcal{M} ; \beta} \in d \gamma\right) \propto \exp \left(-\beta L^{\mathcal{M}}(\gamma)\right) \prod_{e \in \operatorname{Edges}(\gamma)} \mathcal{M}(d l[e]), \quad \gamma \in \Gamma_{D},
$$

where $l[e]$ is the straight line extending $e$. In other words, the probability of having $\mathcal{A}_{D}^{\mathcal{M} ; \beta} \in d \gamma$ is proportional to the Boltzmann factor $\exp \left(-\beta L^{\mathcal{M}}(\gamma)\right)$ times the product of edge activities $\mathcal{M}(d l[e]), e \in \operatorname{Edges}(\gamma)$. Observe also that this construction should be regarded as a specific version of the general polygonal model given in (2.11) of [2]. The finiteness of the partition function

$$
\mathcal{Z}_{D}^{\mathcal{M} ; \beta}:=\mathbb{E} \sum_{\gamma \in \Gamma_{D}\left(\Lambda_{D}^{\mathcal{M}}\right)} \exp \left(-\beta L^{\mathcal{M}}(\gamma)\right)<\infty
$$

for all $\beta \in \mathbb{R}$ is not difficult to verify and has been established in [16], see (32) there.

In this paper we shall focus on polygonal fields in their consistent regime corresponding to $\beta=1$. As shown in Sect. 3 in [16], this particular choice of temperature parameter places us in the context of a non-homogeneous version of Arak-Surgailis [2] construction for the consistent polygonal fields, see Sect. 4 there. This ensures striking properties of the field. First of these, the consistency, states that for each open bounded and convex $D \subseteq D^{\prime} \subset \mathbb{R}^{2}$ the field $\mathcal{A}_{D}^{\mathcal{M}}:=\mathcal{A}_{D}^{\mathcal{M} ; 1}$ coincides in law with $\mathcal{A}_{D^{\prime}}^{\mathcal{M}}:=\mathcal{A}_{D^{\prime}}^{\mathcal{M} ; 1} \cap D$, thus allowing for a direct construction of the infinite volume process (thermodynamic limit) $\mathcal{A}^{\mathcal{M}}:=\mathcal{A}^{\mathcal{M} ; 1}$ on the whole $\mathbb{R}^{2}$ such that $\mathcal{A}_{D}^{\mathcal{M}}=\mathcal{A}^{\mathcal{M}} \cap D$. The infinite volume process $\mathcal{A}^{\mathcal{M}}$ takes its values in the space $\Gamma:=\Gamma_{\mathbb{R}^{2}}$ of whole-plane admissible configurations, with obvious meaning of this notation. Further, the explicit formula for the partition function $\mathcal{Z}_{D}^{\mathcal{M}}$ is known for convex $D$, see Theorem 4.1 in [2] for the homogeneous case and Theorem 1 in [16] for the general non-homogeneous set-up. We state this formula in (6). Moreover, one-dimensional linear sections of the field are fully characterised in distribution, see ibidem and Theorem 2 in [16]. Finally, the polygonal fields $\mathcal{A}_{D}^{\mathcal{M}}$ enjoy the two-dimensional Markov property stating that the conditional behaviour of the field inside a smooth closed curve depends on the outside configuration only through arbitrarily small neighbourhoods of the curve or, equivalently, through the trace of the external configuration on the curve, consisting of intersection points and directions. This property is a direct consequence of the Gibbsian definition $(2,3)$ of the field and, unlike the previous properties, it holds for all inverse temperatures $\beta \in \mathbb{R}$ rather than just for $\beta=1$. We will not discuss this property in the present paper and we refer the reader to the original work of Arak and Surgailis [2] for further details.

\section{Generalised Dynamic Representation for Consistent Fields}

The present section is meant to recall the generalised dynamic representation for consistent polygonal fields as developed in Sect. 4 of [16], which will serve as a crucial tool in our further considerations. The name generalised representation comes from the fact that it 
generalises the original construction of such fields introduced by Arak and Surgailis in [2]. In the sequel we will often omit the qualifier generalised for the sake of terminological brevity. To describe the representation, fix the convex field domain $D$ and let $\left(D_{t}\right)_{t \in[0,1]}$ be a time-indexed increasing family of compact convex subsets of $\bar{D}$, eventually covering the entire $\bar{D}$ and interpreted as a growing window gradually revealing increasing portions of the polygonal field under construction in the course of the time flow. In other words, under this interpretation, the portion of a polygonal field in a bounded open convex domain $D$ uncovered by time $t$ is precisely its intersection with $D_{t}$. To put it in formal terms, consider $\left(D_{t}\right)_{t \in[0,1]}$ satisfying

(D1) $\left(D_{t}\right)_{t \in[0,1]}$ is a strictly increasing family of compact convex subsets of $\bar{D}=D \cup \partial D$.

(D2) $D_{0}$ is a single point $x$ in $\bar{D}=D \cup \partial D$.

(D3) $D_{1}$ coincides with $\bar{D}$.

(D4) $D_{t}$ is continuous in the usual Hausdorff metric on compacts.

Note that the extra fifth condition imposed on $D_{t}$ in Sect. 4 of [16] is automatically satisfied here due to the absolute continuity $\mathcal{M} \ll \mu$ and thus is not mentioned here. Clearly, under these conditions, for $\mathcal{M}$-almost each $l \in[[D]]$ the intersection $l \cap D_{\tau_{l}}$ consists of precisely one point $\mathbb{A}(l)$, where $\tau_{l}=\inf \left\{t \in[0,1], D_{t} \cap l \neq \emptyset\right\}$. The point $\mathbb{A}(l)$ will be referred to as the anchor point for $l$, this induces the anchor mapping $\mathbb{A}:[[D]] \rightarrow D$ defined $\mathcal{M}$-almost everywhere. Consider now the following dynamics in time $t \in[0,1]$, with all updates given by the rules below performed independently of each other.

(GE:Initialise) Begin with empty field at time 0,

(GE:Unfold) Between critical moments listed below, during the time interval $[t, t+d t]$ the unfolding field edges in $D_{t}$ reaching $\partial D_{t}$ extend straight to $D_{t+d t} \backslash D_{t}$,

(GE:BoundaryHit) When a field edge hits the boundary $\partial D$, it stops growing in this direction (note that $\mathcal{M}$-almost everywhere the intersection of a line with $\partial D$ consists of at most two points),

(GE:Collision) When two unfolding field edges intersect in $D_{t+d t} \backslash D_{t}$, they are not extended any further beyond the intersection point (stop growing in the direction marked by the intersection point),

(GE:DirectionalUpdate) A field edge extending along $l \in\left[\left[D_{t}\right]\right]$ updates its direction during $[t, t+d t]$ and starts unfolding along $l^{\prime} \in\left[\left[l^{[t, t+d t]}\right]\right]$, extending away from the anchor point $\mathbb{A}\left(l^{\prime}\right)$, with probability $\mathcal{M}\left(d l^{\prime}\right)$, where $l^{[t, t+d t]}:=l \cap\left(D_{t+d t} \backslash D_{t}\right)$. Directional updates of this type are all performed independently,

(GE:LineBirth) Whenever the anchor point $\mathbb{A}(l)$ of a line $l$ falls into $D_{t+d t} \backslash D_{t}$, the line $l$ is born at the time $t$ at its anchor point with probability $\mathcal{M}(d l)$, whereupon it begins extending in both directions with the growth of $D_{t}$ (recall that $l$ is $\mathcal{M}$-almost always tangential to $\partial D_{t}$ here),

(GE:VertexBirth) For each intersection point of lines $l_{1}$ and $l_{2}$ falling into $D_{t+d t} \backslash D_{t}$, the pair of field lines $l_{1}$ and $l_{2}$ is born at $l_{1} \cap l_{2}$ with probability $\mathcal{M}\left(d l_{1}\right) \mathcal{M}\left(d l_{2}\right)$, whereupon both lines begin unfolding in the directions away from their respective anchor points $\mathbb{A}\left(l_{1}\right)$ and $\mathbb{A}\left(l_{2}\right)$.

Observe that the evolution rule (GE:VertexBirth) means that pairs of lines are born at birth sites distributed according to a Poisson point process in $D$ with intensity measure given by the intersection measure $\langle\langle\mathcal{M}\rangle\rangle$ of $\mathcal{M}$ defined as follows

$$
\langle\langle\mathcal{M}\rangle\rangle(A):=\frac{1}{2} \mathcal{M} \times \mathcal{M}\left(\left\{\left(l_{1}, l_{2}\right), l_{1} \cap l_{2} \subset A\right\}\right) .
$$




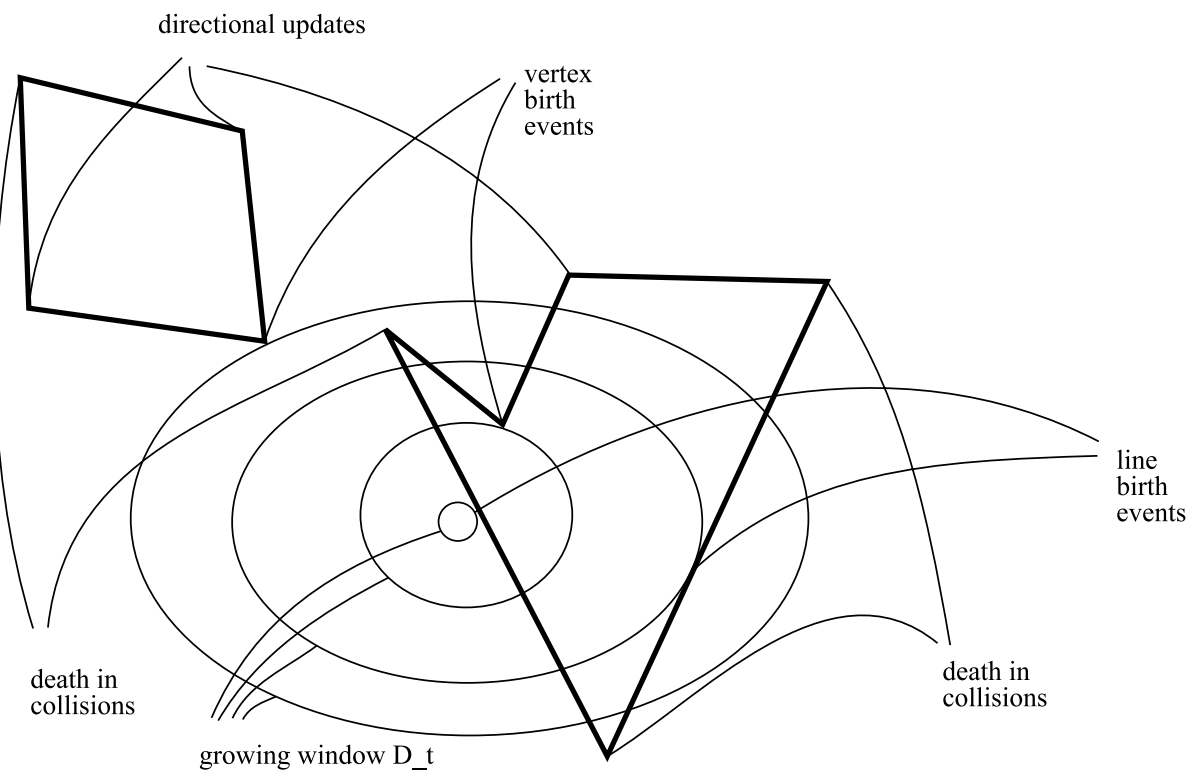

Fig. 2 The generalised dynamic representation for polygonal fields

The importance of the intersection measure lies in the fact that

$$
\mathcal{Z}_{D}^{\mathcal{M} ; 1}=\exp (\langle\langle\mathcal{M}\rangle\rangle(D))
$$

as shown in Theorem 1 in [16]. The following result stating that the polygonal field resulting from the above construction actually coincides with $\mathcal{A}_{D}^{\mathcal{M}}$ has been established in [16], see Theorem 3 there.

Theorem 1 The random contour ensemble resulting from the above construction $(\boldsymbol{G E})$ coincides in law with $\mathcal{A}_{D}^{\mathcal{M}}$.

A typical realisation of the generalised dynamic representation is depicted in Fig. 2.

\section{Polygonal Web}

Having defined the non-homogeneous polygonal fields and presented their graphical construction, we pass now to another object central to this paper, which we name the polygonal $w e b$. Whereas the details of the connection between the critical polygonal web and the corresponding consistent polygonal field are to be established in the subsequent Sect. 5, here we emphasise that, in a sense, the polygonal web constitutes the dual object to the polygonal field sharing the same activity measure, and this duality is going to be reflected in the construction of the polygonal web. Roughly speaking, the polygonal web arises as the union of critical branching polygonal random walks, interacting by an additional bridge-creating mechanism, clasping the branched polygonal trees into a web. 


\subsection{Constructing the Polygonal Web}

Consider an open bounded and convex domain $D$ and let $\left(D_{t}\right)_{t \in[0,1]}$ be a growing family of compact subsets of $\bar{D}$ satisfying the usual conditions (D1-4) as in Sect. 3. Further, assume a collection $(\bar{l}, \bar{x})=\left(l_{i}, x_{i}\right)_{i=1}^{k}$ is given where $x_{i}$ are points in $\bar{D}$ whereas $l_{i}$ are straight lines with $x_{i} \in l_{i}, i=1, \ldots, k$. Moreover, to avoid uninteresting pathologies we require that no three different lines $l_{i}, i=1, \ldots, k$, intersect at one point. Each such pair $\left(l_{i}, x_{i}\right)$ will be called an edge marker because $l_{i}$ can be interpreted as a directional indicator for a linear segment/edge passing through $x_{i}$, see Sect. 5 below where this interpretation is further developed and exploited. The entire collection $(\bar{l}, \bar{x})$ will be referred to as the edge marker configuration. The polygonal web $\mathcal{W}[(\bar{l}, \bar{x})]:=\mathcal{W}_{D}^{\mathcal{M}}[(\bar{l}, \bar{x})]$ generated by $(\bar{l}, \bar{x})$ in $\bar{D}$, with activity measure $\mathcal{M}$, is the union of polygonal trees in $\bar{D}$ arising as the final state $w_{1}$ of the following graphical construction process $w_{s}$ evolving for $s \in[0,1]$, where all random updates listed are performed independently.

(W:Start) At the time $s=0$ we let $w_{0}$ consist of zero-length edges (edge germs) at $x_{i}$, $i=1, \ldots, k$, directed along the respective $l_{i}$ 's.

(W:GrowInwards) Between the critical moments listed below, during the time interval $[s, s+d s]$ all edges of $w_{s}$ reaching the boundary $\partial D_{1-s}$ extend straight to $D_{1-s} \backslash D_{1-s-d s}$ along their respective directional lines. The edges (edge germs) not yet touched by the boundary of the shrinking domain $D_{1-s}$ (and hence contained in the interior of $D_{1-s}$ ) remain intact and do not evolve until eventually hit by the boundary at some later time (unless they get frozen prior to that, see below, in which case they never start evolving). Below, we call edges reaching the current boundary $\partial D_{1-s}$ active and we say that edges (edge germs) not yet hit by the boundary are inactive. Note that the intersection point of a web edge with the current boundary $\partial D_{1-s}$ can be interpreted as its instantaneous growth-tip and so will it be called in the sequel. Observe that inactive edges do not have growth-tips.

(W:BranchAndTurn) During the time interval $[s, s+d s]$ an active web edge reaching the boundary $\partial D_{1-s}$ and extending along $l \in\left[\left[D_{1-s}\right]\right]$ yields a new offspring edge starting at $l^{[s, s+d s]}:=l \cap D_{1-s} \backslash D_{1-s-d s}$ and directed along $l^{\prime} \in\left[\left[l^{[s, s+d s]}\right]\right]$ with probability $\mathcal{M}\left(d l^{\prime}\right)$. Both the original and offspring edges go on evolving according to the usual rules. The branching updates are performed independently for all active web edges.

(W:ForcedBranchAndTurn) If during the time interval $[s, s+d s]$ an active web edge extending along some $l \in\left[\left[D_{1-s}\right]\right]$ and reaching the boundary $\partial D_{1-s}$ intersects the directional line $l^{\prime}$ of some other non-frozen web edge in $w_{s} \cap D_{1-s}$, be it active or inactive, and there is no web edge along $l^{\prime}$ reaching $l \cap \partial D_{1-s}$ and created in a prior stage of the evolution, then a new offspring edge is created at $l \cap \partial D_{1-s}$ directed along $l^{\prime}$ and both the original and offspring edges go on evolving according to the usual rules. Note that we only consider directional lines $l^{\prime}$ of edges hitting the domain $D_{1-s}$ - the edges present in $\bar{D} \backslash D_{1-s}$ but terminated before the time $s$ (see below for edge termination events) are not taken into account. Thus, the forced branching occurs if the growth-tip of an edge hits the current directional line of another (non-frozen) edge currently present in the system. Whenever a forced branching occurs, the resulting offspring edge is called a forced edge whereas the edge whose directional line $l^{\prime}$ gave rise to the branching is referred to as the corresponding forcing edge. Observe that a pair of forced and forcing edges will meet and coalesce into a single edge at further stages of the construction unless one of the edges terminates prior to that.

Note that if the forcing edge is active, it resides at the boundary of the domain $D_{s}$ and yields a single forced edge on the opposite side of the domain. On the other hand, an 
inactive forcing edge (edge germ) located in the interior of $D_{s}$ may give rise to (at most) two forced edges, one on each side of the domain.

(W:Terminate) During the time interval $[s, s+d s]$ an active web edge reaching the boundary $\partial D_{1-s}$ and extending along $l \in\left[\left[D_{1-s}\right]\right]$ terminates (stops evolving) with probability $\mathcal{M}\left(\left[\left[l^{[s, s+d s]}\right]\right]\right)$ where $l^{[s, s+d s]}:=l \cap D_{1-s} \backslash D_{1-s-d s}$.

(W:StopIfSeparated) Whenever at some time moment $s \in[0,1]$ a web edge (or edge germ) $e$ in $w_{s} \cap D_{1-s}$, be it active or inactive, has the property that $l[e] \cap \operatorname{conv}\left(\left[w_{s} \backslash\right.\right.$ $\left.e] \cap \partial D_{1-s}\right)=\emptyset$ (that is to say the directional line $l[e]$ of the web edge $e$ does not hit the convex hull generated by the current growth-tips of the remaining non-terminated active edges and non-frozen germination points of the remaining inactive edges, in which case we say that $e$ separates from $w_{s}$ at the time $s$ ), then $e$ terminates and stops evolving at this point. Note that in case of an inactive edge germ $e$ to terminate means to remain frozen in inactive state and never to activate even when hit by the boundary at the later stages of the evolution.

A careful reader might ask at this moment why in the above construction we do not consider the case when at some time $s$ a web edge becomes tangential to the domain $D_{1-s}$. The answer is that, with probability one, such cases do not occur in the course of the construction because an edge to become tangential to the boundary of the domain separates from the web prior to that and thus gets terminated by an application of (W:StopIfSeparated) rule.

The construction of the polygonal web as presented above admits a natural intuitive description - the edge germs initiating the process emit critical branching polygonal random walks directed by the activity measure $\mathcal{M}$ and unfolding inward the domain $D$ which can be regarded as dual to the dynamic representation in Sect. 3 where the growth was directed outwards. The branching is critical because the binary branching and termination intensities coincide in (W:BranchAndTurn) and (W:Terminate). The role of the additional (W:ForcedBranchAndTurn) rule is to ensure the possibility of bridging the gaps between two separated co-linear parts of the same segment present on two opposite sides of the window $D_{1-s}$, thus clasping the polygonal trees into a web. Finally, as may be seen in the sequel, the (W:StopIfSeparated) rule reflects the structural knowledge on independence of edge covering events in polygonal fields as established in [16], and as such it is not indispensable in its full form for the theory developed below to be valid and may be replaced by various weaker variants, see Remark 1.

It is useful to note that, regarded as a polygonal graph, the polygonal web contains Tshaped nodes (branching points), I-shaped nodes (edge terminal points) and X-shaped nodes (edge intersection points) but no V-shaped nodes.

Example In Fig. 3 we present a typical realisation of a polygonal web. For clarity, we take $\bar{D}=D_{1}$ to be a rectangle there, $D_{0}$ to be a point on its left side and we assume that $D_{s}$ unfolds as follows: during some initial period $[0, \epsilon]$ the window $D_{s}$ grows along the left side of $\bar{D}$, covering it completely by the time $\epsilon$. Thereupon, $D_{s}$ starts growing as a rectangular window in $\bar{D}$ sharing its left side. This goes by letting a vertical line sweep from left to right in $\bar{D}$ and making it the right side for $D_{s}$. The upper and lower sides of $D_{s}$ are then the respective segments of the upper and lower sides of $\bar{D}$. Recall that in the construction of the polygonal web the time flows backwards and the window $D_{s}$ shrinks from $D_{1}$ to $D_{0}$ which corresponds to the inverse right-to-left vertical line sweep in our Fig. 3.

\subsection{Crop Functional}

To establish a direct link between the polygonal web and polygonal fields we define now the crop functional of the polygonal web, further denoted as $\operatorname{crop}(\mathcal{W}[(\bar{l}, \bar{x})])$. To this end, 


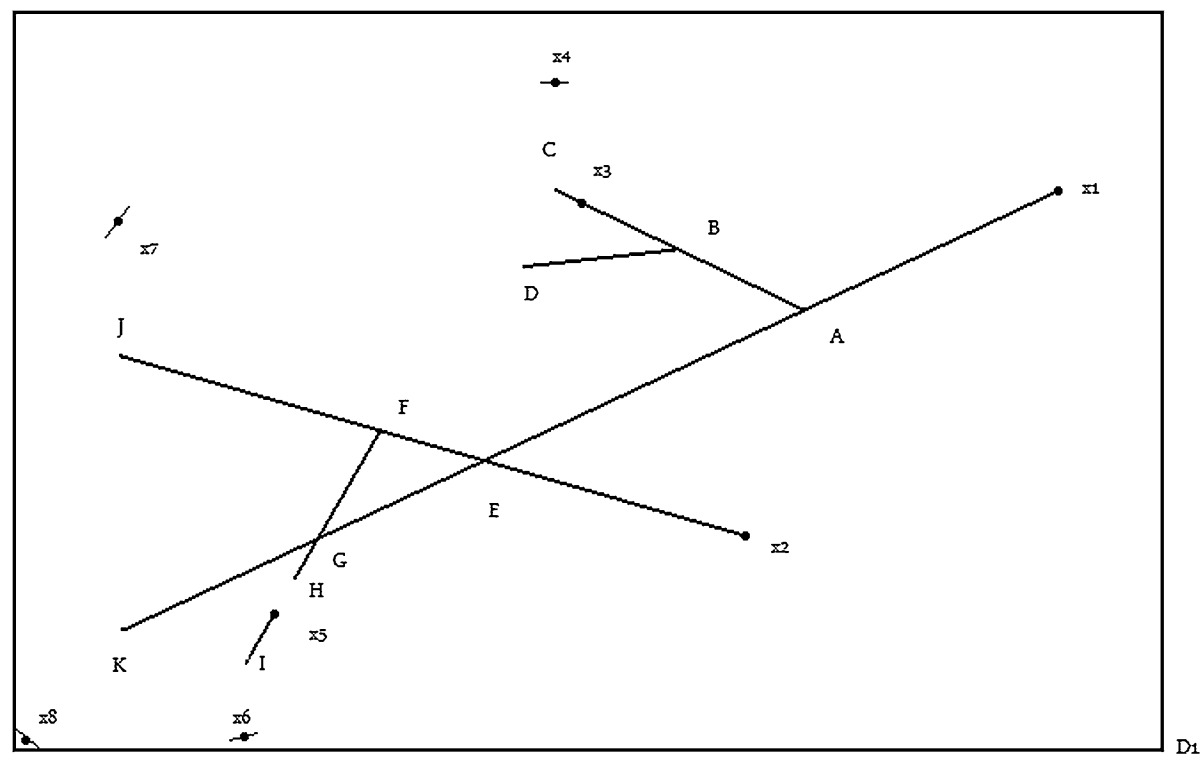

Fig. 3 The construction of polygonal web. At the point $A$ a forced branching occurs to form a bridge to $x_{3}$. Next, at $B$ a non-forced branching takes place. The bridge $\overline{A x_{3}}$ successfully reaches $x_{3}$ but thereafter the evolution of this branch stops at $C$ due to separation. The branch starting at $x_{4}$ terminates immediately at its birth, again due to separation. Next, at $D$ the branch terminates by (W:Terminate). At $E$ two branches cross but nothing happens. Further, at $F$ a forced branching occurs in an attempt to bridge towards $x_{5}$. Although passing through $G$ and even going a bit further, this attempt is unsuccessful as terminating at $H$ by (W:Terminate) and thus leaving an incomplete bridge. The branch emitted from $x_{5}$ stops at $I$ by separation. The remaining two branches stop at $J$ and $K$ by separation. The branches at $x_{6}, x_{7}$ and $x_{8}$ terminate immediately upon birth, by separation. The short segments at $x_{4}, x_{6}, x_{7}$ and $x_{8}$ are not actual edges of the polygonal web and their purpose is only to indicate the respective directions $l_{4}, l_{6}, l_{7}$ and $l_{8}$

we identify the polygonal web $\mathcal{W}[(\bar{l}, \bar{x})]=\mathcal{W}_{D}^{\mathcal{M}}[(\bar{l}, \bar{x})]$ with the collection of branches connecting the initial edge germ locations $x_{i}, i=1, \ldots, k$ to the edge termination points $y_{1}, \ldots, y_{m}$ resulting from (W:Terminate,StopIfSeparated) rules or arising as meeting points where forcer-forced pairs of co-linear web edges unfolding in opposite directions merge into linear segments. To avoid nuisance technicalities below we formally interpret each meeting point of a forcer-forced pair as two distinct points, one terminating the forcing branch, the second terminating the forced branch. Clearly, $m \geq k$ since each initial edge germ $x_{i}$ emits at least one branch and each branch eventually terminates. Keeping $(\bar{l}, \bar{x})$ fixed, we shall index the branches constituting $\mathcal{W}[(\bar{l}, \bar{x})]$ by their terminal points, writing branch $\left[y_{j}\right]$ for the branch terminating at $y_{j}$-the inambiguity of this indexation is ensured by the above convention on meeting points of forcer-forced pairs. Along each branch we have a natural chronological ordering from the root $x_{i}, i \in\{1, \ldots, k\}$ to the endpoint $y_{j}$, $j \in\{1, \ldots, m\}$. For a collection of branches branch $[y], y \in \mathcal{Y}, \mathcal{Y} \subseteq\left\{y_{1}, \ldots, y_{m}\right\}$, we consider the induced polygonal crop graph CropGraph[Y] obtained as follows.

(Crop:Grow) Follow the growth of all branches in $\{\operatorname{branch}[y], y \in \mathcal{Y}\}$ starting from their roots and unfolding towards their respective endpoints during the time interval $[0,1]$ as in the course of the polygonal web dynamics $(\mathbf{W})$.

(Crop:StopOnCollision) Whenever in the course of their growth two branches meet, they both stop growing at this point. 
Thus, the crop graph is a subgraph of $\mathcal{W}[(\bar{l}, \bar{x})]$ containing T-shaped, I-shaped and V-shaped nodes but not $\mathrm{X}$-shaped nodes. This is because the crop graph arises by (recursively) cutting off the parts of branches past their intersections with other branches present in the inducing collection. There are two ways in which two branches can meet in the above constructionthey can either intersect coming from two non-colinear directions and giving rise to a $\mathrm{V}$ shaped node in CropGraph[Y] , or meet coming from opposite co-linear directions yielding a linear segment rather than a graph node. Note that two distinct branches sharing a common sub-branch and thus coinciding during initial growth phase are not considered to meet or intersect! On the other hand, if several distinct branches coinciding during an initial growth phase intersect another branch(es) during this phase, the growth-interrupting (Crop:StopOnCollision) rule applies to all these branches simultaneously.

It is easily seen that two different collections of terminal points can yield identical crop graphs because some branch turning points can be cut off due to collisions in the course of the (Crop) dynamics. It is clear though that for each instance of a crop graph arising in (Crop) dynamics there exists a unique collection of terminal points with the property that no branch turning points occur past the cut-off points. This unique collection is called minimal for its crop graph, or just minimal for short if no ambiguity arises.

To proceed, we say that a collection $\mathcal{Y} \subseteq\left\{y_{1}, \ldots, y_{m}\right\}$ of branch-determining endpoints is complete iff each initial germ location is the root of some branch $[y], y \in \mathcal{Y}$. Clearly, the cardinality of such a complete collection cannot fall below $k$. Further, we say that the crop graph CropGraph[Y] of a complete endpoint collection $\mathcal{Y}$ is normal iff it contains no forced edges which fail to eventually meet and merge with their co-linear forcing edges. Note that this condition can be violated by either having a forced edge without its forcer present in the graph or due to a death or directional update along either a forcing or a forced edge. Whereas the death of either edge in an forcer-forced pair does necessarily lead to the lack of normality, a directional update does so only if there is no other branch in the collection along which the considered forcer-forced pair could extend further past the turning point. A graph which is not normal is called abnormal. Thus, roughly speaking, an abnormal graph is a graph containing the forced part of an incomplete bridge. Write

$$
\iota(\mathcal{Y}):= \begin{cases}1, & \text { if } \mathcal{Y} \subseteq\left\{y_{1}, \ldots, y_{m}\right\} \text { is complete, minimal and CropGraph[Y] is normal } \\ 0, & \text { otherwise. }\end{cases}
$$

With this notation, we define the crop functional

$$
\operatorname{crop}(\mathcal{W}[(\bar{l}, \bar{x})]):=\sum_{\mathcal{Y} \subseteq\left\{y_{1}, \ldots, y_{m}\right\}}(-1)^{\operatorname{card}(\mathcal{Y})-k} \iota(\mathcal{Y})
$$

The expression (8) has the aesthetic advantage of defining the crop functional in a form reminiscent of the classical inverse Möbius transform, with the summation performed over all subsets of $\left\{y_{1}, \ldots, y_{m}\right\}$, see e.g. Sect. 2.6 in [12]. To exploit this feature define

$$
\hat{\imath}(\mathcal{Y}):= \begin{cases}1, & \text { if CropGraph }[\mathcal{Y}] \text { is normal } \\ 0, & \text { otherwise }\end{cases}
$$

that is to say $\hat{\imath}$ is the indicator of crop graph normality, without the extra completeness and minimality requirements. Given a crop graph $\varrho=\operatorname{CropGraph}[\mathcal{Y}]$ for some complete and minimal $\mathcal{Y}_{\varrho} \subseteq\left\{y_{1}, \ldots, y_{m}\right\}$, we let $B[y], y \in \mathcal{Y}_{\varrho}$, be the set of all $y_{j}, j=1, \ldots, m$, such that branch $\left[y_{j}\right]$ contains the entire subbranch branch $[y] \cap \varrho$. Then, for any $\mathcal{Y} \subseteq\left\{y_{1}, \ldots, y_{m}\right\}$ 
such that CropGraph[Y] $=\operatorname{CropGraph}\left[\mathcal{Y}_{\varrho}\right]$ we have $\hat{\imath}(\mathcal{Y})=\iota\left(\mathcal{Y}_{\varrho}\right)$ and, moreover, $\mathcal{Y}$ decomposes into the disjoint union of non-empty $\mathcal{Y}_{y}=\mathcal{Y} \cap B[y], y \in \mathcal{Y}_{\varrho}$. We also have $\operatorname{card}(\mathcal{Y})=\operatorname{card}\left(\mathcal{Y}_{\varrho}\right)+\sum_{y \in \mathcal{Y}_{\varrho}}\left[\operatorname{card}\left(\mathcal{Y}_{y}\right)-1\right]$. Consequently, using Newton's binomial formula,

$$
\begin{aligned}
& \sum_{\mathcal{Y}, \text { CropGraph }[\mathcal{Y}]=\operatorname{CropGraph}\left[\mathcal{Y}_{\varrho}\right]}(-1)^{\operatorname{card}(\mathcal{Y})-k} \hat{\iota}(\mathcal{Y}) \\
& =(-1)^{\operatorname{card}\left(\mathcal{Y}_{\varrho}\right)-k} \iota\left(\mathcal{Y}_{\varrho}\right) \prod_{y \in \mathcal{Y}_{\varrho}} \sum_{\emptyset \neq \mathcal{Y}_{y} \subseteq B[y]}(-1)^{\operatorname{card}\left(\mathcal{Y}_{y}\right)-1}=(-1)^{\operatorname{card}\left(\mathcal{Y}_{\varrho}\right)-k} \iota\left(\mathcal{Y}_{\varrho}\right) \prod_{y \in \mathcal{Y}_{\varrho}} 1 \\
& =(-1)^{\operatorname{card}\left(\mathcal{Y}_{\varrho}\right)-k} \iota\left(\mathcal{Y}_{\varrho}\right) .
\end{aligned}
$$

Thus, (8) can be alternatively rewritten as

$$
\operatorname{crop}(\mathcal{W}[(\bar{l}, \bar{x})])=\sum_{\mathcal{Y} \subseteq\left\{y_{1}, \ldots, y_{m}\right\}, \mathcal{Y} \text { complete }}(-1)^{\operatorname{card}(\mathcal{Y})-k} \hat{\imath}(\mathcal{Y}) .
$$

Further, we put $T\left[x_{i}\right]:=\left\{y_{j} \in\left\{y_{1}, \ldots, y_{m}\right\}, \operatorname{root}\left(\operatorname{branch}\left[y_{j}\right]\right)=x_{i}\right\}, i=1, \ldots, k$, and write, applying Newton's binomial formula,

$$
\sum_{\mathcal{Y} \subseteq\left\{y_{1}, \ldots, y_{m}\right\}, \mathcal{Y}}(-1)^{\operatorname{card}(\mathcal{Y})-k}=\prod_{i=1}^{k} \sum_{\emptyset \neq \mathcal{Y}_{i} \subseteq T\left[x_{i}\right]}(-1)^{\operatorname{card}\left(\mathcal{Y}_{i}\right)-1}=\prod_{i=1}^{k} 1=1 .
$$

Combining this with (9) we conclude that

$$
\operatorname{crop}(\mathcal{W}[(\bar{l}, \bar{x})])=1-\sum_{\mathcal{Y} \subseteq\left\{y_{1}, \ldots, y_{m}\right\}, \mathcal{Y} \text { complete }}(-1)^{\operatorname{card}(\mathcal{Y})-k}[\hat{\imath}(\mathcal{Y})-1]
$$

and hence the crop equals one for polygonal webs whose all complete branch subcollections yield normal crop graphs, whereas the deviations of the crop functional from the standard value one are due to crop graph abnormalities, which will be further exploited in the sequel.

A natural alternative way of defining the crop functional involves summation over crop subgraphs of $\mathcal{W}[(\bar{l}, \bar{x})]$, that is to say over all possible different graphs arising in the (Crop) dynamics above, in which case (8) becomes

$$
\operatorname{crop}(\mathcal{W}[(\bar{l}, \bar{x})])=\sum_{\varrho \text { is a normal crop graph in } \mathcal{W}[\bar{l}, \bar{x})]}(-1)^{\text {number of branchings in } \varrho}
$$

since the number of branchings in $\varrho=$ CropGraph[Y $], \mathcal{Y}$ minimal, is easily seen to coincide with $\operatorname{card}(\mathcal{Y})-k$. Note that by a branching we understand here a point in the crop graph from which two different branches outgo, thus in other words a branching corresponds to a T-shaped (but not V-shaped) node of the crop graph.

Example In Figs. 4, 5 and 6 below we visualise the concepts of this subsection for the example polygonal web shown in Fig. 3 . Note that the branch-determining endpoints there are $\left\{y_{1}, \ldots, y_{11}\right\}:=\left\{x_{3}, C, x_{4}, D, H, I, x_{6}, J, K, x_{7}, x_{8}\right\}$. Observe also that the point $x_{3}$ has been included as terminating the degenerate forcing branch $\overline{x_{3} x_{3}}$ of the bridge $\overline{x_{3} x_{3}}$.

To get some insight into the structure of the family of complete and minimal endpoint collections yielding normal crop graphs, we note first that among the possible endpoints 


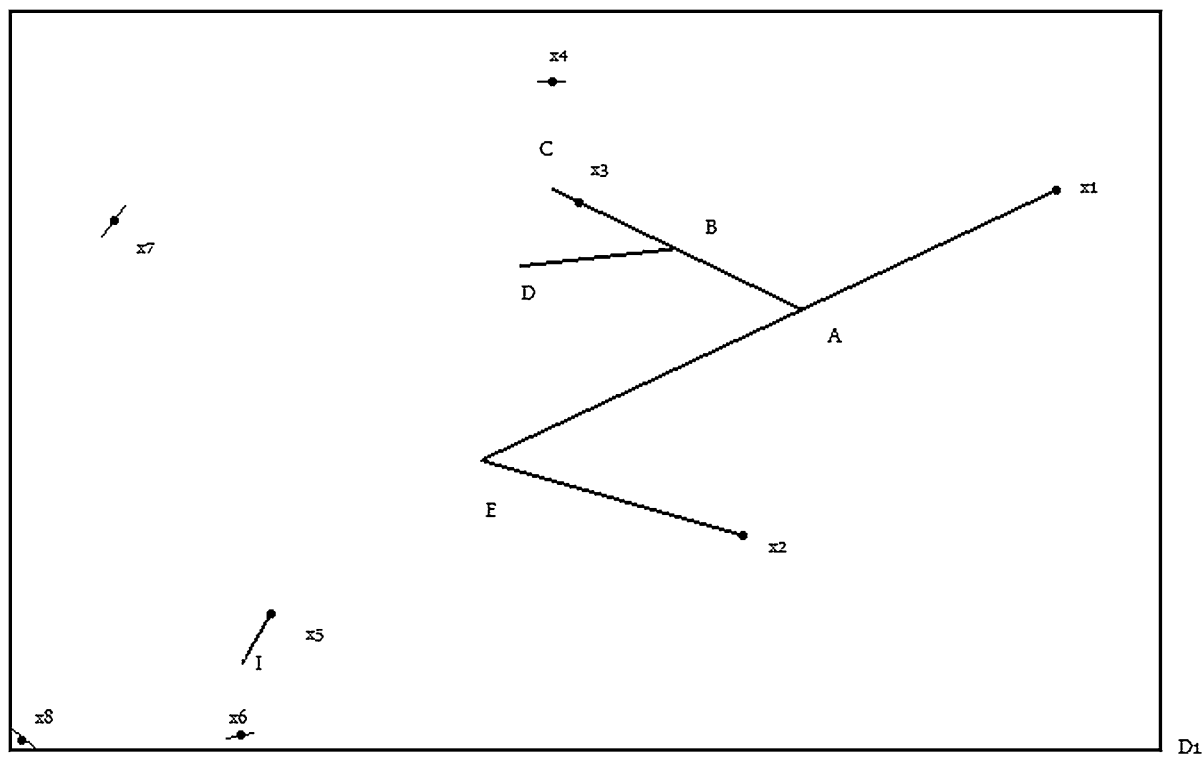

Fig. 4 The crop graph for $\left\{x_{3}, C, x_{4}, D, I, x_{6}, J, K, x_{7}, x_{8}\right\}$. This is a complete and minimal collection and the corresponding graph is normal. To make the collection non-minimal we could augment it with $H$

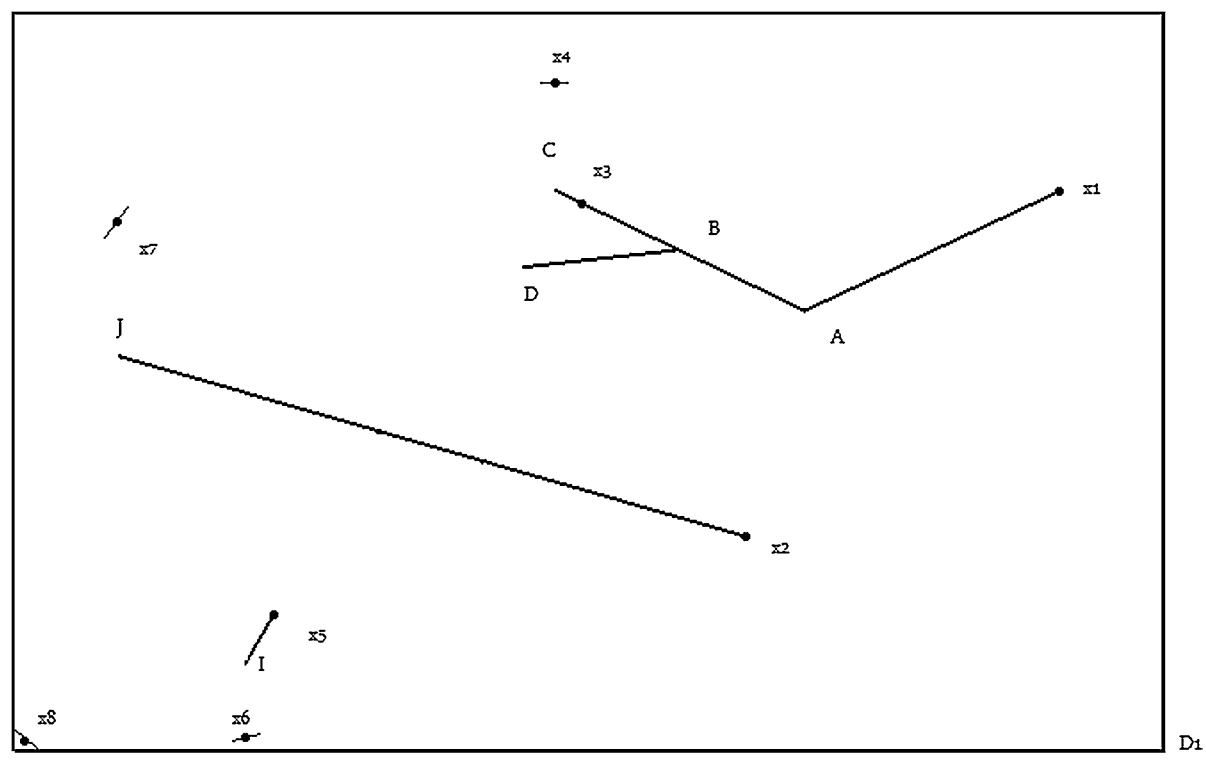

Fig. 5 The crop graph for $\left\{x_{3}, C, x_{4}, D, I, x_{6}, J, x_{7}, x_{8}\right\}$. This is a complete and minimal collection and the corresponding graph is normal

$\left\{x_{3}, C, x_{4}, D, H, I, J, K, x_{7}, x_{8}\right\}$ points $C, x_{4}, I, x_{6}, x_{7}, x_{8}$ always have to be picked for completeness. Next, $H$ cannot be picked because it always produces an incomplete bridge (unless the collection is non-minimal). However, this means in turn that also $J$ has to be picked 


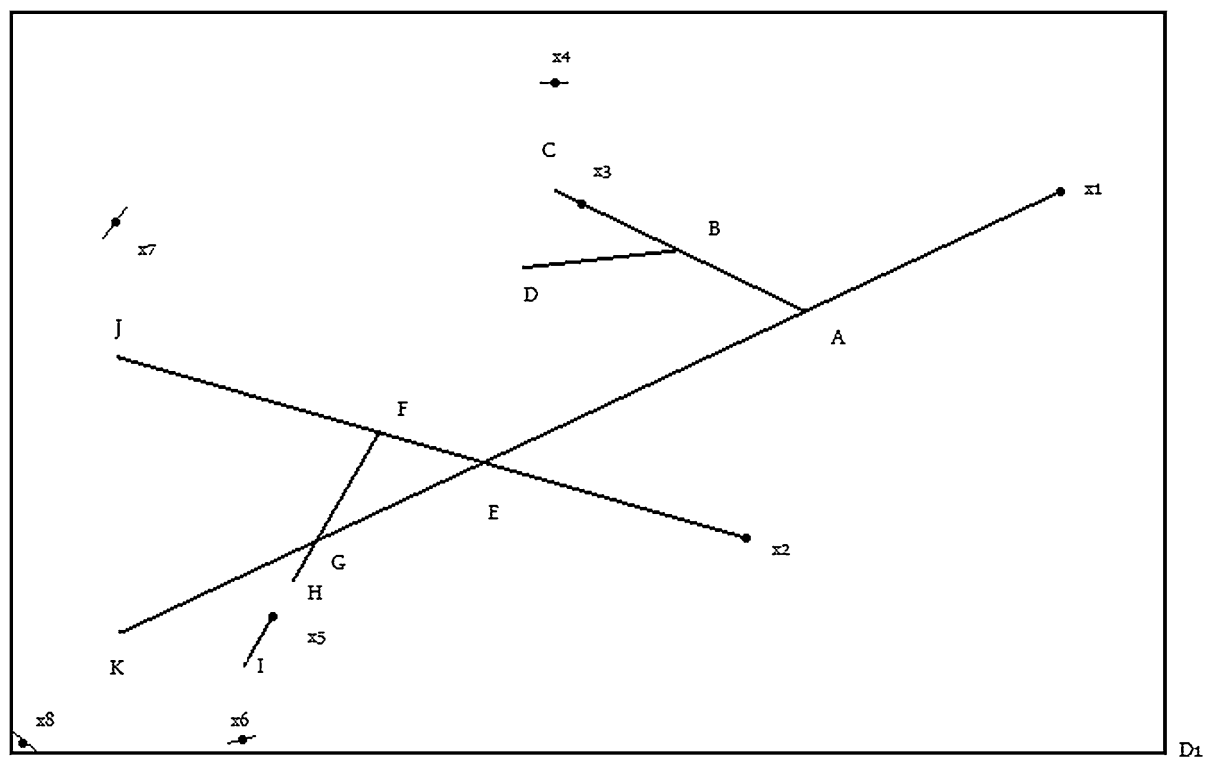

Fig. 6 The crop graph for $\left\{x_{3}, C, x_{4}, D, H, I, x_{6}, J, x_{7}, x_{8}\right\}$. This is a complete and minimal collection but the corresponding graph is not normal because it contains an incomplete bridge terminating at $H$

to preserve completeness, because $H$ was its only alternative to include $x_{2}$ as a branch root. Moreover, we cannot take $D$ if $x_{3}$ is not picked, as it would result in a gap along the bridge $\overline{A x_{3}}$. Furthermore, if neither $x_{3}$ nor $D$ are taken then $K$ has to be taken to ensure that a branch outgrows from $x_{1}$. Otherwise, all choices are permitted, which eventually leaves us with 5 options for complete and minimal $\mathcal{Y}$ giving rise to a normal crop graph:

1. $\mathcal{Y}=\left\{x_{3}, C, x_{4}, D, I, x_{6}, J, K, x_{7}, x_{8}\right\}$, CropGraph $(\mathcal{Y})$ has 2 branchings.

2. $\mathcal{Y}=\left\{x_{3}, C, x_{4}, D, I, x_{6}, J, x_{7}, x_{8}\right\}$, CropGraph $(\mathcal{Y})$ has 1 branching.

3. $\mathcal{Y}=\left\{x_{3}, C, x_{4}, I, x_{6}, J, K, x_{7}, x_{8}\right\}$, CropGraph $(\mathcal{Y})$ has 1 branching.

4. $\mathcal{Y}=\left\{x_{3}, C, x_{4}, I, x_{6}, J, x_{7}, x_{8}\right\}$, CropGraph $(\mathcal{Y})$ has no branchings.

5. $\mathcal{Y}=\left\{C, x_{4}, I, x_{6}, J, K, x_{7}, x_{8}\right\}$, CropGraph $(\mathcal{Y})$ has no branchings.

Using (11) yields now $\operatorname{crop}\left(\mathcal{W}[(\bar{l}, \bar{x})]=1\right.$ for the considered collection $\left(l_{i}, x_{i}\right)_{i=1}^{8}$.

Clearly, calculations of this sort are somewhat tedious when made by hand, but they can be easily carried out by a computer. As already mentioned in the introduction, the theory developed in this paper will be used to construct an exact Bayesian inference engine in the space of polygonal shapes, specialised to the hybrid semi-discrete set-up of [17] with a view towards image processing applications. This is a subject of our ongoing work in progress and will be published in a separate paper.

\subsection{Interpretation of the Crop Functional}

A few words are due at this point to provide an intuitive interpretation of the crop functional as formally defined in (8). To this end, we begin by mentioning a kid game quite popular in the happy time of the author's childhood: given a collection of arrows on a sheet of paper draw a family of closed curves passing through these arrows, and in case where this can be done in more than one way resolve the ambiguity by trying to make the resulting picture 
resemble some real-life object. In fact, this and related problems are not just games and find serious interest in studies on human and computer vision, see [5] and the references therein. In mathematical terms and specialising to our polygonal set-up, given an edge marker collection $(\bar{l}, \bar{x})=\left(l_{i}, x_{i}\right)_{i=1}^{k}$ we ask for admissible polygonal configurations $\gamma \in \Gamma_{\mathbb{R}^{2}}$ with the following properties

- Each edge $e$ of $\gamma$ contains some edge marker point $x_{i(e)}$ such that $l[e]=l_{i(e)}$.

- Each edge marker point $x_{i}$ is contained in some $e(i) \in \operatorname{Edges}(\gamma)$ such that $l[e(i)]=l_{i}$.

Following Sect. 5 of [16] we denote the family of such configurations by $\Gamma(\bar{l}, \bar{x})$ and write $N(\bar{l}, \bar{x})$ for the cardinality of $\Gamma(\bar{l}, \bar{x})$, that is to say the number of solutions to the discussed problem. To proceed, consider first a particularly simple deterministic instance $\mathcal{W}^{0}[(\bar{l}, \bar{x})]:=\mathcal{W}_{D}^{0}[(\bar{l}, \bar{x})]$ of polygonal web generated by $(\bar{l}, \bar{x})$ not depending on the activity measure $\mathcal{M}$-define $\mathcal{W}^{0}[(\bar{l}, \bar{x})]$ to arise in the course of the above $(\mathbf{W})$ dynamics without non-forced turns/branchings and without termination events, which is a usual situation for example when $x_{i}$ 's are very close to each other and are all contained in a domain $D$ with very small $\mathcal{M}([[D]])$ where applications of (W:TurnAndBranch,Terminate) are very unlikely. Observe that the notation $\mathcal{W}_{D}^{0}[(\bar{l}, \bar{x})]$ comes from the fact that the above construction of this polygonal web coincides with the (W) dynamics under zero activity measure. Then, as will be shown in Lemma 2 below,

$$
\operatorname{crop}\left(\mathcal{W}_{D}^{0}[(\bar{l}, \bar{x})]\right)=N(\bar{l}, \bar{x})
$$

for each bounded convex domain $D$ containing all $x_{i}$ 's.

At the other extremity lies the typical behaviour of the crop functional of polygonal webs generated by marker configurations $(\bar{l}, \bar{x})$ where the distances between all $x_{i}$ 's are huge and where all $l_{i}$ 's are pairwise different-due to the criticality of our branching mechanism the usual situation then is that the web $\mathcal{W}[(\bar{l}, \bar{x})]$ splits into disjoint and distant sub-webs originating from respective $x_{i}$ 's and the bridge creating attempts between these sub-webs fail with overwhelming probability in consequence of (W:Terminate). In these circumstances all normal crop graphs in $\mathcal{W}[(\bar{l}, \bar{x})]$ are readily seen to be unions of disjoint normal crop graphs in sub-webs stemming from individual $x_{i}$ 's and thus, by (11), the value of $\operatorname{crop}(\mathcal{W}[(\bar{l}, \bar{x})])$ factorises into the product of crops of the sub-webs. Using (15) below combined with our main Theorem 2 and resorting to Remark 1 allows us to conclude easily but not immediately that the expectations of these individual crops are all 1 and consequently

$$
\mathbb{E} \operatorname{crop}(\mathcal{W}[(\bar{l}, \bar{x})]) \approx 1
$$

as well. This fact, not studied in detail here, is intimately related to mixing properties of polygonal fields (asymptotic factorisation of edge correlations) but this link will not be followed any further in this paper because so far we are only able to establish a slow polynomial mixing using a rather technical argument based on polygonal webs, whereas alternative methods developed in Sect. 7 of [16] allowed us to establish exponential mixing there at least for rectangular fields.

In remaining situations the crop functional interpolates between the above extremities.

\section{Polygonal Web Representation for Edge Correlations}

The purpose of this section is to formulate our main result stating that arbitrary order edge correlation functions of the polygonal field coincide with the expectations of the respective polygonal web crops. 
Edge Correlations Having introduced the crucial concepts in preceding sections we are now in a position to define the principal object of our study in this paper, that is to say the edge-correlation functions for polygonal fields, and to formulate our main results. Due to the polygonal nature of the considered field the natural object to consider are the edge correlations

$$
\sigma^{\mathcal{M}}\left[d l_{1}, x_{1} ; \ldots ; d l_{k}, x_{k}\right]:=\mathbb{P}\left(\forall_{i=1}^{k} \exists_{e \in \operatorname{Edges}\left(\mathcal{A}^{\mathcal{M}}\right)} \pi_{l_{i}}\left(x_{i}\right) \in e, l[e] \in d l_{i}\right),
$$

where $l_{1}, \ldots, l_{k}$ are straight lines and $\pi_{l_{i}}$ is the orthogonal projection on $l_{i}$. In all cases below we shall be interested in correlations with $x_{i} \in l_{i}$, in which case $\sigma^{\mathcal{M}}\left[d l_{1}, x_{1} ; \ldots ; d l_{k}, x_{k}\right]$ can be interpreted as the probability element that the polygonal field $\mathcal{A}^{\mathcal{M}}$ passes through points $x_{i}$ in the directions determined by the respective lines $l_{i}, i=1, \ldots, k$. For general $x_{i}$, not necessarily lying on $l_{i}$, the $k$-fold correlation $\sigma^{\mathcal{M}}\left[d l_{1}, x_{1} ; \ldots ; d l_{k}, x_{k}\right]$ is the probability that the polygonal field passes through points $\pi_{l_{i}}\left(x_{i}\right)$ in the directions determined by the respective lines $l_{i}, i=1, \ldots, k$.

Recall from Sect. 4 above that collections $(\bar{l}, \bar{x})=\left(l_{i}, x_{i}\right)_{i=1}^{k}$ of lines $l_{i}$ and points $x_{i}$ with $x_{i} \in l_{i}$ are referred to as edge marker configurations (collections) whereas each pair $\left(l_{i}, x_{i}\right)$ belonging to such a collection is called an edge marker. No edge marker can occur twice in an edge marker collection. An edge along $l_{i}$ passing through $x_{i}$ is said to cover the marker $\left(l_{i}, x_{i}\right)$. We say that an edge marker collection $(\bar{l}, \bar{x})=\left(x_{i}, l_{i}\right)_{i=1}^{k}$ is in general position if the lines $l_{i}$ are pairwise different and $x_{j} \notin l_{i}$ for $j \neq i$, otherwise if $l_{i}=l_{j}$ for some $i \neq j$ then the collection is called degenerate and $x_{i}, x_{j}$ are declared coupled by $l_{i}=l_{j}$, finally if $x_{i} \in l_{j}$ for some $i \neq j$ with $l_{i} \neq l_{j}$ then the collection is said to be in singular position. Thus, an edge marker collection can be simultaneously degenerate and singular. As mentioned above, if two edge marker lines $l_{i}=l_{j}$ in a degenerate configuration coincide, we say that the edge markers $\left(l_{i}, x_{i}\right)$ and $\left(l_{j}, x_{j}\right)$ are coupled, sometimes for brevity we just say that $x_{i}$ and $x_{j}$ are coupled. While allowing both for singularity and degeneracy of marker collections, we strictly exclude the situations where three or more different marker lines intersect at one point, in order to avoid unnecessary technical pathologies.

Note that in the singular case where $x_{i} \in l_{j}$ for some $i \neq j$ but $l_{i} \neq l_{j}$ it makes sense to consider one-sided correlations $\sigma^{\mathcal{M}}\left[d l_{i}^{+}, x_{i} ; d l_{j}, x_{j} ; \ldots\right]$ and $\sigma^{\mathcal{M}}\left[d l_{i}^{-}, x_{i} ; d l_{j}, x_{j} ; \ldots\right]$ with $l_{i}^{+}$and $l_{i}^{-}$standing for two half-lines into which $l_{i}$ is split by the intersection point $\left\{x_{i}\right\}=$ $l_{i} \cap l_{j}$. The definition of such correlations is analogous to (12) the difference being that the field edge containing $x_{i}$ is required to extend respectively at least in the direction of $l_{i}^{+}$and $l_{i}^{-}$, yet it is also allowed although not required to extend in the opposite direction as well. Since $\mathcal{M} \ll \mu$, it is easily seen that for a configuration in general position we would have $\sigma^{\mathcal{M}}\left[d l_{i}^{+}, x_{i} ; d l_{j}, x_{j} ; \ldots\right]=\sigma^{\mathcal{M}}\left[d l_{i}^{-}, x_{i} ; d l_{j}, x_{j} ; \ldots\right]=\sigma^{\mathcal{M}}\left[d l_{i}, x_{i} ; d l_{j}, x_{j} ; \ldots\right]$, but this is no more the case in the considered singular situation, where the respective correlations are non-trivially affected by the event that an edge along $l_{j}$ may extend from $x_{j}$ down to $x_{i}$ where it may intersect with another edge along $l_{i}$.

In the sequel we will use the notation $\Delta^{*}[k], * \in\{g, s, d\}$, for the respective sets of all collections $\left(l_{i}, x_{i}\right)_{i=1}^{k}$ in general, singular and degenerate positions. Further, we put $\Delta^{*}:=$ $\bigcup_{k=1}^{\infty} \Delta^{*}[k], * \in\{g, s, d\}$ and $\Delta[k]:=\bigcup_{* \in\{g, s, d\}} \Delta^{*}[k]$ and $\Delta:=\bigcup_{* \in\{g, s, d\}} \Delta[k]$. Clearly, $\Delta[k]$ can be endowed with the natural product topology from $\left(\left[\left[\mathbb{R}^{2}\right]\right] \times \mathbb{R}^{2}\right)^{\times k}$.

Edge Correlation Functions For a non-degenerate edge marker configuration $(\bar{l}, \bar{x})=$ $\left(l_{i}, x_{i}\right)_{i=1}^{k}$ the (normalised) $\mathrm{k}$-fold edge correlation function $\phi\left(l_{1}, x_{1} ; \ldots ; l_{k}, x_{k}\right)$ is defined by

$$
\phi\left(l_{1}, x_{1} ; \ldots ; l_{k}, x_{k}\right)=\phi^{\mathcal{M}}\left(l_{1}, x_{1} ; \ldots ; l_{k}, x_{k}\right):=\frac{\sigma^{\mathcal{M}}\left[d l_{1}, x_{1} ; \ldots ; d l_{k}, x_{k}\right]}{\mathcal{M}\left(d l_{1}\right) \ldots \mathcal{M}\left(d l_{k}\right)}
$$


More generally, if $(\bar{l}, \bar{x})$ is a degenerate edge marker configuration, its normalised correlation is given by

$$
\phi(\bar{l}, \bar{x})=\frac{\sigma^{\mathcal{M}}\left[d l_{1}, x_{1} ; \ldots ; d l_{k}, x_{k}\right]}{\prod^{*} \mathcal{M}\left(d l_{i}\right)},
$$

where $\prod^{*}$ stands for the product over lines $l_{i}$ in which each line is present exactly once, with repetitions discarded. We also adopt the convention that $\phi(\emptyset):=1$, where $\emptyset$ stands for empty edge marker configuration. The existence of edge correlation functions is guaranteed by the following lemma.

Lemma 1 The correlation function $\phi(\bar{l}, \bar{x})$ exists for each edge marker configuration $(\bar{x}, \bar{l})$ and is continuous on $\Delta^{g}[k]$. In particular, $\phi(\cdot)$ is locally bounded.

Likewise, we can also consider one-sided versions of the correlation functions, for which the existence and local boundedness statements of Lemma 1 readily extend. Note that in general the correlation functions are not continuous at singular configurations in $\Delta^{s}[k]-$ indeed, if a sequence of configurations in general positions converges to some singular configuration, which implies that some $x_{i}$ asymptotically reaches $l_{j}$ with $j \neq i$, the limit of the respective correlation functions can be easily seen to coincide with the appropriate onesided correlation function for the limit singular configuration, provided the convergence of $x_{i}$ takes place on one side of $l_{j}$ only, otherwise the limit may fail to exist. The discontinuity may therefore arise because the one-sided correlation functions may differ on different sides.

By Theorem 4 in [16] it follows that for all $(\bar{l}, \bar{x}) \in \Delta^{g}[k], k=1,2$, we have

$$
\phi(\bar{l}, \bar{x})=1
$$

The same paper gives general conditions for this relation to hold for $k>2$. Here we are interested in the general set-up for $k>2$ where it often happens that $\phi(\bar{l}, \bar{x}) \neq 1$.

Representation Theorem for Edge Correlation Functions The following theorem is the main result of this paper.

Theorem 2 For each edge marker collection $(\bar{l}, \bar{x})$ with $\bar{x} \subset D$ we have

$$
\phi^{\mathcal{M}}(\bar{l}, \bar{x})=\mathbb{E} \operatorname{crop}\left(\mathcal{W}_{D}^{\mathcal{M}}[(\bar{l}, \bar{x})]\right) .
$$

It is useful at this point to compare Theorem 2 with the simple observation that whenever all $x_{i}$ 's in $(\bar{l}, \bar{x})$ belong to a small ball $B_{2}\left(x_{0}, r\right)$ for some $x_{0} \in \mathbb{R}^{2}$ then

$$
\phi(\bar{l}, \bar{x})=N(\bar{l}, \bar{x})(1+O(r)),
$$

with $N(\bar{l}, \bar{x})$ defined as in Sect. 4.3, which readily follows by $(2,3)$ and the definitions $(13,14)$ of edge correlation functions, taking additionally into account the local boundedness of the density $m(\cdot)$ of the activity measure $\mathcal{M}$ with respect to the Haar-Lebesgue measure $\mu$ which ensures that the Boltzmann factors $\exp \left(-L^{\mathcal{M}}(\cdot)\right)$ are $1+O(r)$ on $B_{2}\left(x_{0}, r\right)$, see also (12) in [16]. It is interesting to note at this point that, by the consistency of $\mathcal{A}^{\mathcal{M}}$, the same would hold if we defined $N(\bar{x}, \bar{l})$ to be the cardinality of $\Gamma(\bar{x}, \bar{l}) \cap D$ for any convex 
open $D \supset \bar{x}$ and thus $N(\bar{x}, \bar{l})$ does not depend on the field domain $D$ as long as $D \supset \bar{x}$ ! To proceed, observe that under the same conditions our Theorem 2 yields

$$
\phi(\bar{l}, \bar{x})=\operatorname{crop}\left(\mathcal{W}_{D^{r}}^{0}[(\bar{l}, \bar{x})]\right)(1+O(r))
$$

with $\mathcal{W}_{D^{r}}^{0}[(\bar{l}, \bar{x})]$ defined as in Sect. 4.3 and where $D^{r}$ is an r-dependent bounded convex domain of diameter $O(r)$ containing $B_{2}\left(x_{0}, r\right)$. This is because $O(r)$ is the probability that at least one turning/branching event occurs in the course of the polygonal web generating dynamics $(\mathbf{W})$ confined to $D^{r}$, again in view of the local boundedness of the density $m(\cdot)$. To proceed we note that both $N(\bar{l}, \bar{x})$ and $\operatorname{crop}\left(\mathcal{W}_{D^{r}}^{0}[(\bar{l}, \bar{x})]\right)$ are, by their definitions, invariant with respect to non-singular affine transforms of $(\bar{l}, \bar{x})$. Consequently, upon an appropriate re-scaling we can take $r$ in (16) and (17) arbitrarily small. This way, upon comparing (16) and (17) we have established

Lemma 2 For each edge marker collection $(\bar{l}, \bar{x})$ we have

$$
\operatorname{crop}\left(\mathcal{W}_{D}^{0}[(\bar{l}, \bar{x})]\right)=N(\bar{l}, \bar{x})
$$

for each $D \supset \bar{x}$. In particular, $\operatorname{crop}\left(\mathcal{W}_{D}^{0}[(\bar{l}, \bar{x})]\right)$ does not depend on $D \supset \bar{x}$.

Note at this point that the relation (17) can be further extended to produce a small $r$ expansion, with the coefficient at $r^{k}$ corresponding to instances of polygonal webs with exactly $k$ turns/branchings. We do not pursue this topic here though because we are not aware of any natural geometric interpretations for the higher order terms of this expansion in the style of Lemma 2.

Relaxing the Stop if Separated Rule As has already been remarked above, in contrast to the remaining rather strict dynamic rules, the (W:StopIfSeparated) rule can be somewhat relaxed without affecting the validity of our Theorem 2 . This is made more specific in the remark below.

Remark 1 A direct inspection of the proof of our representation Theorem 2 below shows that the result stays valid if the (W:StopIfSeparated) rule, requiring the web branch growth to cease immediately when its tip separates from the remaining ones, get replaced some other rule where the growth is stopped only on separation but not necessarily immediately at separation. The only natural constraints are that

- At each time moment in the course of the graphical construction the decision on whether to stop the growth of a separated branch either depends deterministically on the present configuration of branches or at least it is independent of the future evolutions of branches given the current branch configuration.

- Each branch eventually dies before or at the moment $s$ of becoming tangential to the current domain boundary $\partial D_{s}$.

The first condition precludes unwanted dependencies whereas the second one is indispensable for the technical correctness of our constructions (the above tangency point is the point where the time flow direction changes along a branch, and the growth only occurs forward in time in our constructions).

Note that a particular simple example of a stopping rule satisfying the above conditions is to kill each branch exactly at the time $s$ when it becomes tangential to the current boundary $\partial D_{s}$. 
In fact, an even deeper analysis of our argument below shows that further relaxation of the considered rule are admissible. We do not discuss these details in this paper though as they are of no use for our present purposes.

\section{Proof of the Representation Theorem 2}

The purpose of this section is to prove our main Theorem 2. Our argument splits into several parts and requires some additional concepts.

Edge Marker Process To proceed towards establishing our representation theorem for edge correlation functions, we shall introduce a Markovian edge marker process whose construction can be to some extent regarded as a backwards version of the dynamic representation discussed in Sect. 3. Roughly speaking, the dynamic representation involved an explosion of the field from a single point up to the entire domain $D$, whereas the edge marker process represents an evolution back in time and thus an implosion of the marker configuration, eventually to reach one of possible null states. In fact, the edge marker process will be seen to encode the construction of the polygonal web, see (18) below, providing an interpolation between the original marker configuration $(\bar{l}, \bar{x})$ and the full polygonal web $\mathcal{W}[(\bar{l}, \bar{x})]$, whence the backwards time flow direction. To make all this specific, take the increasing family $\left(D_{t}\right)_{t \in[0,1]}$ of convex compacts satisfying (D1-4) as chosen in Sect. 4. Next, consider a continuous time branching edge marker process $\Psi_{s}:=\Psi_{s ; D}, s \in[0,1]$, taking its values in finite families of signed and possibly empty edge marker configurations, with generic notation

$$
\Psi_{s}=\left\{\eta^{(p)}:\left(\bar{l}^{(p)}(s), \bar{x}^{(p)}(s)\right)=\left(l_{i}^{(p)}(s), x_{i}^{(p)}(s)\right)_{i=1}^{k_{p}}\right\}_{p=1}^{m}
$$

with $m$ and $k_{p}$ allowed to depend on the time $s$ and where $\eta^{(p)} \in\{+1,-1\}$. As the notation suggests, the signs $\eta^{(p)}$ are attributed once and for all to their respective marker configurations and do not evolve in time. In addition, we always require that

(EM:DomainShrink) for each $s \in[0,1]$ the marker points $x_{i}^{(p)}(s)$ are all contained in the set $D_{1-s}$,

that is to say the domain of the process $\Psi_{s}$ shrinks over time as informally discussed above. Given the initial state $\Psi_{0}$ with all marker points contained in $\bar{D}=D_{1}$ and, in addition, assumed not to contain three different edge marker lines meeting at one point, the process $\Psi_{s}$ is governed by the following Markovian dynamics (EM), clearly preserving the latter property in view of the absolute continuity $\mathcal{M} \ll \mu$.

(EM:DiscardIfSeparated) If at some time $s$ an edge marker $\left(l_{i}^{(p)}(s), x_{i}^{(p)}(s)\right)$ has the property that $l_{i}^{(p)}(s)$ does not hit the convex hull

$$
C_{i}^{(p)}(s):=\operatorname{conv}\left(\left\{x_{j}^{(q)}(s), q=1, \ldots, m ; j=1, \ldots, k_{q}\right\} \backslash\left\{x_{j}^{(p)}(s)\right\}\right)
$$

generated by all the remaining $x_{j}^{(q)}(s)$ 's in all marker configurations $\left(\bar{l}^{(q)}(s), \bar{x}^{(q)}(s)\right), q=$ $1, \ldots, m$, in which case we say that the marker $\left(l_{i}^{(p)}(s), x_{i}^{(p)}(s)\right)$ separates from $\Psi_{s}$, then remove the marker $\left(l_{i}^{(p)}(s), x_{i}^{(p)}(s)\right)$ from its configuration $\left(\bar{l}^{(p)}(s), \bar{x}^{(p)}(s)\right)$.

If the removal of $\left(l_{i}^{(p)}(s), x_{i}^{(p)}(s)\right)$ makes some other markers separate from $\Psi_{s}$, the (EM:DiscardIfSeparated) rule applies for them as well and they are subsequently removed. 
(EM:FoldInwards) Between the critical moments listed below, at each time $s \geq 0$ each edge marker point $x_{i}^{(p)}(s)$ lying at the boundary $\partial D_{s}$-in which case we say the marker is in boundary position - moves along its corresponding marker line $l_{i}^{(p)}(s)$ so as to always stay at the boundary of the shrinking domain, that is to say $x_{i}^{(p)}(s+d s)$ arises as the intersection of $l_{i}^{(p)}(s)$ with $\partial D_{s+d s}$. Note that marker points not lying at the boundary $\partial D_{s}$ do not move until they are eventually met by the boundary at some later time.

(EM:DiscardOnCollision) If at some time $s$ two edge marker points $x_{i}^{(p)}(s)$ and $x_{j}^{(p)}(s)$ within the same marker collection $\left(\bar{l}^{(p)}(s), \bar{x}^{(p)}(s)\right)$ collide (meet) along non-colinear directions $l_{i}^{(p)}(s)$ and $l_{j}^{(p)}(s)$, the two markers are removed from the configuration.

As a result of the above (EM:DiscardOnCollision) rule some other edge markers may separate from $\Psi_{s}$-in such a case the (EM:DiscardIfSeparated) rule applies immediately and the markers get discarded.

(EM:Kill) In the course of the time interval $[s, s+d s]$, an edge marker $\left(l_{i}^{(p)}(s), x_{i}^{(p)}(s)\right)$ in boundary position, moving along the segment $x_{i}^{(p)}[s, s+d s]:=\overline{x_{i}^{(p)}(s) x_{i}^{(p)}(s+d s)}$, gets removed from its configuration $\left(\bar{l}^{(p)}(s), \bar{x}^{(p)}(s)\right)$ with probability $\mathcal{M}\left(\left[\left[x_{i}^{(p)}[s, s+d s]\right]\right]\right)$. These updates are performed independently for all different boundary edge markers throughout all marker configurations constituting the process $\Psi_{s}$, yet they are performed $s i$ multaneously for all equal edge markers contained in different configurations, that is to say if $\left(x_{i}^{(p)}(s), l_{i}^{(p)}(s)\right)=\left(x_{j}^{(q)}(s), l_{j}^{(q)}(s)\right)$ for some $p \neq q$ then the kill events during $[s, s+d s]$ coincide for both these markers. In other words, the killing mechanism is a.s. identical for all instances of an edge marker present in different configurations constituting $\Psi_{s}$.

As a result of the above (EM:Kill) rule some other edge markers may separate from $\Psi_{s}-$ in such a case the (EM:DiscardIfSeparated) rule applies immediately and the markers get discarded.

(EM:TurnAndBranch) In the course of the time interval $[s, s+d s]$, for each boundary edge marker $\left(l_{i}^{(p)}(s), x_{i}^{(p)}(s)\right)$ moving along the corresponding segment $x_{i}^{(p)}[s, s+d s]:=$ $\overline{x_{i}^{(p)}(s) x_{i}^{(p)}(s+d s)}$, with probability $\mathcal{M}(d l)$ for $l \in\left[\left[x_{i}^{(p)}[s, s+d s]\right]\right]$ a turn-and-branch update occurs in the direction of $l$, which results in replacing the original marker configuration $\left(\bar{l}^{(p)}, \bar{x}^{(p)}\right)$ by three offspring marker configurations in $\Psi_{s}$, which are:

- $\eta^{(p)}:\left(\bar{l}^{(p)}(s), \bar{x}^{(p)}(s)\right)$ (unmodified offspring),

- $\eta^{(p)}:\left(\bar{l}^{(p)}(s), \bar{x}^{(p)}(s)\right) \backslash\left\{\left(l_{i}^{(p)}(s), x_{i}^{(p)}(s)\right)\right\} \cup\left\{\left(l_{i}^{(p)}(s+d s):=l, x_{i}^{(p)}(s+d s)\right)\right\}$ (directional update offspring-the original marker line $l_{i}^{(p)}(s)$ turns in the direction of $l$, that is to say $\left.l_{i}^{(p)}(s+d s):=l\right)$,

- $-\eta^{(p)}:\left(\bar{l}^{(p)}(s), \bar{x}^{(p)}(s)\right) \cup\left\{\left(l, x_{i}^{(p)}(s+d s)\right)\right\}$ (branched offspring-both the original marker and its directional update are present).

As in the case of (EM:Kill) above, these updates are performed independently for all different boundary edge markers throughout all marker configurations constituting the process $\Psi_{s}$, yet they are performed simultaneously for all equal edge markers contained in different configurations, that is to say if $\left(x_{i}^{(p)}(s), l_{i}^{(p)}(s)\right)=\left(x_{j}^{(q)}(s), l_{j}^{(q)}(s)\right)$ for some $p \neq q$ then the turning/branching updates during $[s, s+d s]$ coincide for both these markers. In other words, the turning/branching mechanism is a.s. identical for all instances of an edge marker present in different configurations constituting $\Psi_{s}$.

Whenever the above (EM:TurnAndBranch) update is performed with $l \cap C_{i}^{(p)}(s+d s)=$ $\emptyset$, which results in the directionally updated edge marker separating from $\Psi_{s}$, the rule (EM:DiscardIfSeparated) applies immediately to the corresponding directional update and branched offsprings. 
(EM:ForcedTurnAndBranch) If a boundary edge marker point $x_{i}^{(p)}(s)$ crosses some edge marker line $l_{j}^{(p)}(s), j \neq i, l_{j}^{(p)}(s) \neq l_{i}^{(p)}(s)$, during the time interval $[s, s+d s]$, a forced turn-and-branch update occurs in the direction of $l_{j}^{(p)}(s)$, which results in replacing the original marker configuration $\left(\bar{l}^{(p)}, \bar{x}^{(p)}\right)$ by three offspring marker configurations in $\Psi_{s}$, which are:

- $\eta^{(p)}:\left(\bar{l}^{(p)}(s), \bar{x}^{(p)}(s)\right)$ (unmodified offspring),

- $\eta^{(p)}:\left(\bar{l}^{(p)}(s), \bar{x}^{(p)}(s)\right) \backslash\left\{\left(l_{i}^{(p)}(s), x_{i}^{(p)}(s)\right)\right\} \cup\left\{\left(l_{i}^{(p)}(s+d s):=l_{j}^{(p)}(s), x_{i}^{(p)}(s+d s)\right)\right\}(\mathrm{di}-$ rectional update offspring - the original marker line $l_{i}^{(p)}(s)$ turns in the direction of $l_{j}^{(p)}(s)$, that is to say $\left.l_{i}^{(p)}(s+d s):=l_{j}^{(p)}(s)\right)$,

- $-\eta^{(p)}:\left(\bar{l}^{(p)}(s), \bar{x}^{(p)}(s)\right) \cup\left\{\left(l_{j}^{(p)}(s), x_{i}^{(p)}(s+d s)\right)\right\}$ (branched offspring-both the original marker and its directional update are present).

It should be noted at this point that, unlike in the usual (EM:TurnAndBranch) discussed above, here (EM:DiscardIfSeparated) has no chance of becoming applicable directly upon the update because along $l_{j}^{(p)}(s)$ there always exists a direction pointing at $C_{i}^{(p)}(s+d s)$, namely that towards $x_{j}^{(p)}(s+d s)$. Moreover, observe also that, in effect of the so-defined (EM:ForcedTurnAndBranch) update, $x_{i}^{(p)}(s+d s)$ and $x_{j}^{(p)}(s+d s)$ become coupled both in the directional update and branched offsprings.

(EM:UnbreakableCouplings) If at some time $s$ in the course of their evolution two marker points $x_{i}^{(p)}(s)$ and $x_{j}^{(p)}(s)$ are coupled in their configuration then whenever they cease to be so in the original configuration or any of its offspring configurations, the coupling-breaker configuration is instantly removed from $\Psi_{s}$. Note that this is equivalent to the rejection of configurations where

- a coupled edge marker gets killed in a collision (EM:DiscardOnCollision) or in (EM:Kill),

- a coupled edge marker modifies its direction in directional update offsprings arising in (EM:TurnAndBranch,ForcedTurnAndBranch).

The unmodified and branched offsprings do not break couplings and neither can a coupling be broken in (EM:DiscardIfSeparated) because coupled markers are never separated since they always point at their pair. Note that in contrast to (EM:DiscardIfSeparated) rule, where we discard individual edge markers, here we remove entire configurations.

If at some time moment a marker point $x_{i}^{(p)}(s)$ reaches its coupled $x_{j}^{(p)}(s)$, both markers coalesce and evolve henceforth as one, in particular all coupling restrictions cease to apply. Such meeting and coalescence may occur at the tangency point of the respective directional line to the current domain $\partial D_{s}$ in which case the resulting single marker is instantly discarded from the system by application of (EM:DiscardIfSeparated).

The above construction of the edge marker process may seem rather bizarre at the first look, but this is in fact a rather simple object. The moving boundary of the shrinking domain $D_{s}$ drives inwards polygonal branching random walks of constituent edge markers. The directional updating and branching mechanisms of these walks are determined by the activity measure $\mathcal{M}$. The directions of the walks are always chosen to point at the convex hull generated by the remaining marker points in the process. If such a choice becomes impossible due to edge marker separation, the marker is discarded. Colliding edge markers are also discarded. One further rule is unbreakability of once established marker couplings, which is ensured by rejecting coupling-breaker configurations. It is important to note that with probability 1 , in the course of the dynamics all markers eventually 
- either separate and are discarded in (EM:DiscardIfSeparated),

- or disappear in collisions (EM:DiscardOnCollision),

- or are killed in (EM:Kill),

- or finally they have their configurations annihilated due to coupling breaks, as an application of (EM:UnbreakableCouplings).

Thus, at time 1 the process $\Psi_{1}$ consists a.s. of signed empty marker configurations.

Another crucial observation, readily verified by comparing the (EM) and (W) dynamics, is that, on the event $\left\{\Psi_{0}=\{+1:(\bar{l}, \bar{x})\}\right\}$, the union of trajectories traced by the constituent marker points $x_{i}^{(p)}(s), s \in[0,1]$, of $\Psi$ coincides with the web $\mathcal{W}[(\bar{l}, \bar{x})]$, that is to say

$$
\mathcal{W}_{D}^{\mathcal{M}}[(\bar{l}, \bar{x})]=\bigcup_{s \in[0,1]} \bigcup_{\left(\bar{l}^{(p)}(s), \bar{x}^{(p)}(s)\right) \in \Psi_{s}} \bigcup_{\left(x_{i}^{(p)}(s), l_{i}^{(p)}\right.} \bigcup_{(s)) \in\left(\bar{l}^{(p)}(s), \bar{x}^{(p)}(s)\right)}\left\{x_{i}^{(p)}(s)\right\} .
$$

Moreover, again by the construction, (the history of) each marker configuration present in $\Psi_{1}$ bijectively corresponds to a complete, minimal and normal collection of branches of $\mathcal{W}[(\bar{l}, \bar{x})]$, whereas the complete abnormal branch collections correspond to marker configurations rejected in (EM:UnbreakableCouplings) (this latter correspondence also becomes a bijection as soon as the minimality of branch collections is assumed).

Recall now our assumption made above stating that the killing, directional updating and branching mechanisms, while independent for different markers, do coincide for equal markers. This assumption is clearly crucial for (18) above to hold, but could be easily lifted without affecting the validity of a significant part of the theory presented below. In fact, these mechanisms can also be coupled in any other way as soon as the Markovian property of the dynamics is preserved. We do not discuss this issue here though as the imposed coupling seems to be the most natural one and leading to simplest formulations.

Some concern may be raised by the branching nature of the (EM) evolution-a natural question is whether no cardinality explosions occur for $\Psi_{s}$. This possibility is easily excluded though, as stated below.

Lemma 3 For each bounded open convex set $D$ and initial condition $\Psi_{0}$ there exists $c\left[D ; \Psi_{0}\right]<+\infty$ such that

$$
\forall_{s \in[0,1]} \quad \mathbb{E} \operatorname{card}\left(\Psi_{s}\right) \leq c\left[D ; \Psi_{0}\right]
$$

To see it use first the relation (18) and the discussion following it to conclude that, for all $s \in[0,1]$, the expectation $\mathbb{E} \operatorname{card}\left(\Psi_{s}\right)$ bounded by $2^{\text {number of branches of } \mathcal{W}[(\bar{l}, \bar{x})]}$, which is the maximum number of possible branch collections. Now, the expectation of this number is finite because $\mathcal{W}[(\bar{l}, \bar{x})]$ arises from a (critical) binary branching process evolving during a finite time interval. Note that the forced branchings do not cause trouble here because they only allow to extend already existing lines born at time $s$ to (at the furthest) the opposite side of $D_{1-s}$, whereas new lines are only born due to the usual critical branching.

Correlation Process Having constructed the edge marker branching process $\Psi_{s}$, we are now going to compose it with the correlation function to obtain the edge correlation process $\Phi_{s}, s \in[0,1]$. We put

$$
\Phi_{s}:=\sum_{\left(\bar{l}^{(p)}(s), \bar{x}^{(p)}(s)\right) \in \Psi_{s}} \eta^{(p)}(s) \phi\left(\bar{l}^{(p)}(s), \bar{x}^{(p)}(s)\right), \quad s \in[0,1],
$$


which means defining the correlation process to be the sum of correlation functions for all marker configurations in $\Psi_{s}$ taken with their corresponding signs. For formal correctness it is convenient to adopt at this point the convention that whenever at some time $s$ the marker point $x_{i}^{(p)}(s)$ is in a singular position lying on some $l_{j}^{(p)}(s), j \neq i$, then the correlation function $\phi\left(\bar{l}^{(p)}(s), \bar{x}^{(p)}(s)\right)$ is interpreted as the one-sided correlation function in which the marker line $l_{i}^{(p)}(s)$ is replaced by half-line $\left[l^{\leftarrow}\right]_{i}^{(p)}(s)$ indicating the direction where $x_{i}^{(p)}(s)$ came from just before hitting $l_{j}^{(p)}(s)$. In view of $(2,3)$ and since $\mathcal{M} \ll \mu$, with probability 1 this is equivalent to putting in such case $\phi\left(\bar{l}^{(p)}(s), \bar{x}^{(p)}(s)\right):=\lim _{u \rightarrow s-} \phi\left(\bar{l}^{(p)}(u), \bar{x}^{(p)}(u)\right)$ for each $s$ where a singularity is reached.

Assume now that $\Psi_{0}=\{+1:(\bar{l}, \bar{x})\}$ and recall from the discussion following the definition of the (EM) dynamics that by the time 1 the marker process $\Psi$ reaches a terminal state consisting entirely of signed empty marker configurations. In view of (18) and the discussion following it, each such empty marker configuration has its history encoded by some complete normal (minimal) branch collection in $\mathcal{W}[(\bar{l}, \bar{x})]$, whereas complete abnormal (minimal) branch collections correspond to marker configurations rejected in (EM:UnbreakableCouplings). Moreover, the sign $\eta$ assigned to each empty marker configuration in $\Psi_{1}$ can be readily verified, by induction in the number of branchings in the course of crop graph creation, to be $(-1)^{\text {number of branchings }}$. Observing that for a complete branch collection, the number of branchings is simply the difference between the number of branches and number of roots (the latter coinciding with the cardinality of the initial marker collection $(\bar{l}, \bar{x}))$ and recalling that $\phi(\emptyset)=1$ we finally conclude from (19) and (8) that

$$
\Phi_{0}=\phi(\bar{l}, \bar{x}), \quad \Phi_{1}=\operatorname{crop}(\mathcal{W}[(\bar{l}, \bar{x})]) .
$$

Martingale Property of the Correlation Process With the notation introduced above, we claim that the edge correlation process is actually a martingale.

Lemma 4 The correlation process $\left(\Phi_{s}\right)_{s \in[0,1]}$ is a martingale with respect to the filtration $\mathcal{F}_{s}$ generated by the marker process $\Psi_{s}$.

In view of the relation (20) Lemma 4 means we have just constructed a martingale interpolating between $(\bar{l}, \bar{x})$ and $\operatorname{crop}(\mathcal{W}[(\bar{l}, \bar{x})])$. This immediately implies the assertion of Theorem 2 upon putting $\Psi_{0}:=\{+1:(\bar{l}, \bar{x})\}$. Thus, it remains to establish the crucial Lemma 4.

Proof of Lemma 4 In view of the Markovian nature of the edge marker process $\Psi_{s}$, to prove Lemma 4 it is enough to establish the desired martingale property at $s=0$. Moreover, for simplicity we present our argument for the initial value $\Psi_{0}$ of the marker process consisting of a single marker configuration $\left(\bar{l}^{(1)}(0), \bar{x}^{(1)}(0)\right):=(\bar{l}, \bar{x})=\left(l_{i}, x_{i}\right)_{i=1}^{k}$, whence the general argument for higher cardinality of the initial condition is readily obtained by straightforward repetition for all configurations in $\Psi_{0}$. We assume without loss of generality that $x_{1}$ lies at the boundary $\partial D_{1}$, for only boundary markers undergo evolution under (EM) dynamics. The remaining marker points $x_{i}, i=2, \ldots, k$ may lie both on $\partial D_{1}$ and in the interior of $D_{1}$.

Using Lemma 3 in [16] and the definition (13) of correlation functions we see that if the line $l_{1}$ does not hit the convex hull conv $\left(\left\{x_{2}, \ldots, x_{k}\right\}\right)$ then

$$
\phi\left(l_{1}, x_{1} ; l_{2}, x_{2} ; \ldots ; l_{k}, x_{k}\right)=\phi\left(l_{2}, x_{2} ; \ldots ; l_{k}, x_{k}\right)
$$


which justifies the (EM:DiscardIfSeparated) rule. Thus, below with no loss of generality we constrain ourselves to the case where the (EM:DiscardIfSeparated) rule does not apply during the period $[0, d s]$ of the (EM) evolution.

To proceed with our argument, we shall use the generalised dynamic representation described in Sect. 3, with the same increasing family of convex compacts $\left(D_{t}\right)_{t \in[0,1]}$ as that used in the construction of the edge marker process. We also recall that $D_{1}=\bar{D}$ where $D$ is the field domain. It should be recalled at this point that the generic time parameter $t$ of the dynamic representation is related by $t=1-s$ to the usual time parameter $s$ of the edge marker and correlation processes. As already signalled above, the idea below is to relate the dynamics of the edge marker process with the graphical representation under inverted (backward) time flow.

If there is another marker point $x_{i}, i \neq 1$, in boundary position with the property that $l_{1}$ and $l_{i}$ meet in $D_{1} \backslash D_{1-d s}$ then, putting $i=2$ for notational clarity, by (13) and the (GE:VertexBirth) dynamic rule we have,

$$
\phi\left(l_{1}, x_{1} ; l_{2}, x_{2} ; l_{3}, x_{3} ; \ldots ; l_{k}, x_{k}\right)=(1+o(1)) \phi\left(l_{3}, x_{3} ; \ldots ; l_{k}, x_{k}\right) .
$$

Clearly, the above event corresponds to marker point collision under (EM) dynamics and thus (22) justifies the (EM:DiscardOnCollision) rule. Keeping this in mind, below we only consider the case where $x_{1}$ does not collide with other marker points during [0,ds].

To proceed, write the correlation-defining event

$$
\mathcal{E}\left[d l_{1}, x_{1} ; \ldots ; d l_{k}, x_{k}\right]:=\left\{\forall_{i=1}^{k} \exists_{e \in \operatorname{Edges}\left(\mathcal{A}^{\mathcal{M}}\right)} x_{i} \in e, l[e] \in d l_{i}\right\}
$$

as the intersection of $\mathcal{E}\left[d l_{1}, x_{1}\right]$ and $\mathcal{E}\left[d l_{2}, x_{2} ; \ldots ; d l_{k}, x_{k}\right]$ where

$$
\mathcal{E}\left[d l_{1}, x_{1}\right]:=\left\{\exists_{e \in \operatorname{Edges}(\mathcal{A} \mathcal{M})} x_{1} \in e, l[e] \in d l_{1}\right\}
$$

and

$$
\mathcal{E}\left[d l_{2}, x_{2} ; \ldots ; d l_{k}, x_{k}\right]:=\left\{\forall_{i=2}^{k} \exists_{e \in \operatorname{Edges}\left(\mathcal{A}^{\mathcal{M}}\right)} x_{i} \in e, l[e] \in d l_{i}\right\} .
$$

We shall also denote by $x_{0}$ the intersection point of the marker line $l_{1}$ with $\partial D_{1-d s}$. With this notation and taking into account that $\mathcal{A}_{D}^{\mathcal{M}}=\mathcal{A}^{\mathcal{M}} \cap D$ arises in the dynamic construction with $\left(D_{t}\right)_{t \in[0,1]}$ as discussed above, we are now in a position to represent $\mathcal{E}\left[d l_{1}, x_{1} ; \ldots ; d l_{k}, x_{k}\right]$ as the union of the following events, disjoint modulo a set of negligible probability, whose names were chosen to represent what happens if we move along the field edge covering the marker $\left(l_{1}, x_{1}\right)$ under the (EM) dynamics inwards $D_{1}$ and towards $x_{0}$.

(E:GoStraight) $\mathcal{E}\left[d l_{2}, x_{2} ; \ldots ; d l_{k}, x_{k}\right]$ occurs and an edge $e_{1}$ along $l_{1}$ covering both $x_{1}$ and $x_{0}$ is present in the field $\mathcal{A}_{D}^{\mathcal{M}}$, see Fig. 7 .

(E:TurnOutwards) $\mathcal{E}\left[d l_{2}, x_{2} ; \ldots ; d l_{k}, x_{k}\right]$ occurs, an edge $e_{1}$ along $l_{1}$ covering $x_{1}$ is present but it does not reach $x_{0}$, instead it turns at some point $x^{\prime}$ of $\overline{x_{1} x_{0}}$ into another field edge $e^{\prime}$ along a line $l^{\prime}$ and in the direction consistent with that determined by the growth of $\left(D_{t}\right)$, that is to say $x^{\prime}$ is the first point of $e^{\prime}$ to be revealed by the growing window $\left(D_{t}\right)$. Note that such an edge $e^{\prime}$ can have only infinitesimally small length within $D_{1}=\bar{D}$ since when moving from $x^{\prime}$ in the direction indicated by the growth of $\left(D_{t}\right)$ we almost immediately encounter the boundary $\partial D$. Thus, we can say that $e^{\prime}$ points outside the domain $\bar{D}$ and away from $D_{1-d s}$, whence the term outward turn. Often in such situations we shall also say that $e^{\prime}$ extends outwards from $x^{\prime}$ along $l^{\prime}$. 
Fig. 7 The event (E:GoStraight)
Fig. 8 The event (E:TurnOutwards)

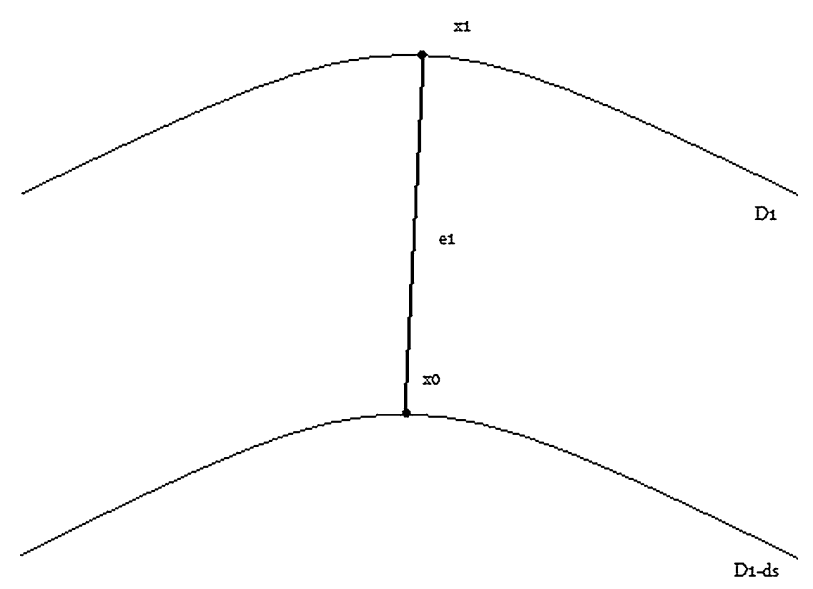

$+\mathrm{E}[\mathrm{dl} 2, \mathrm{x} 2, \ldots, \mathrm{dlk}, \mathrm{xk}]$

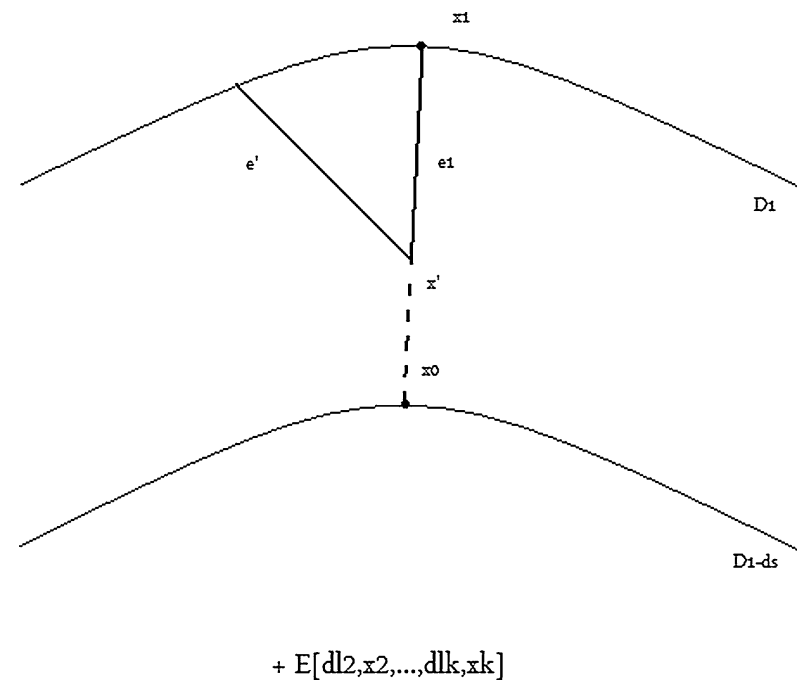

In terms of the dynamic representation the occurrence of (E:TurnOutwards) is equivalent to the occurrence of a (GE:VertexBirth) vertex birth event at $x^{\prime}$ between dynamic representation times $1-d s$ and 1 , giving rise to the edges $e_{1}$ along $l_{1}$ and $e^{\prime}$ along $l^{\prime}$, see Fig. 8.

(E:TurnInwards) $\mathcal{E}\left[d l_{2}, x_{2} ; \ldots ; d l_{k}, x_{k}\right]$ occurs, an edge $e_{1}$ along $l_{1}$ covering $x_{1}$ is present but it does not reach $x_{0}$, instead it turns at some point $x^{\prime}$ of $\overline{x_{1} x_{0}}$ into another field edge $e^{\prime}$ along a line $l^{\prime}$ and in the direction opposite to that determined by the growth of $\left(D_{t}\right)$, that is to say $x^{\prime}$ is the last point of $e^{\prime}$ to be revealed by the growing window $\left(D_{t}\right)$. In contrast to the above outward turn, here we turn in the opposite inward direction. In terms of the dynamic representation the occurrence of (E:TurnInwards) is equivalent to the occurrence of a (GE:DirectionalUpdate) at $x^{\prime}$ where $e^{\prime}$ extending along $l^{\prime}$ turns into $e_{1}$ along $l_{1}$. 
Fig. 9 The event (E:TurnInwards)

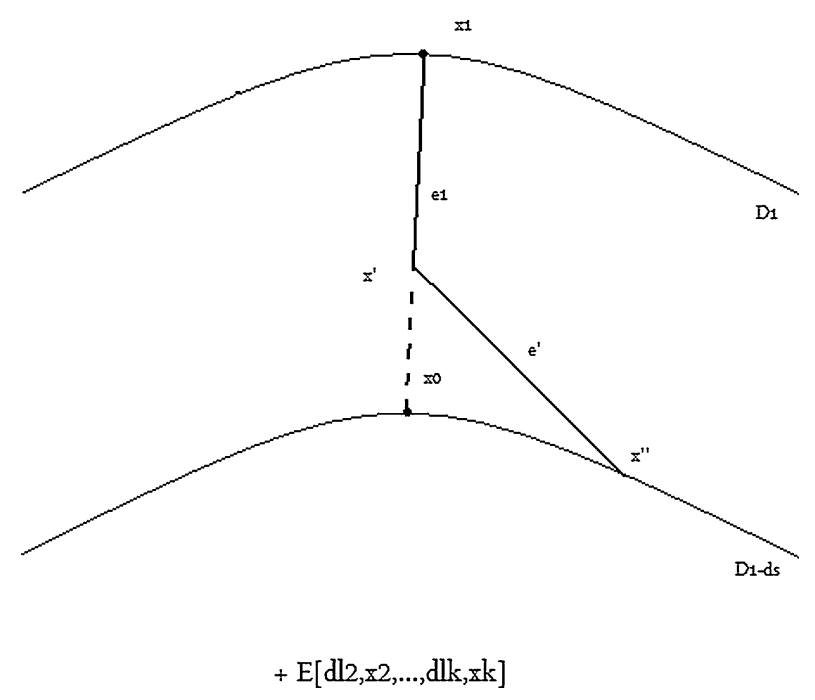

For our considerations below it is convenient at this point to denote by $x^{\prime \prime}$ the intersection point of the inwards half-line $e^{\prime \rightarrow}$ (starting at $x^{\prime}$ and determined by $e^{\prime}$ ) and $\partial D_{1-d s}$. We say that $x^{\prime \prime}$ lies on $l^{\prime}$ inwards from $x^{\prime}$ in such cases. Clearly, $x^{\prime \prime} \in e^{\prime}$ with probability $1-o(1)$. See Fig. 9 for a pictorial representation.

Observe now that, by the dynamic representation,

- For (E:GoStraight) we only consider the case that $x_{1}$ does not collide with any other marker point as the converse case has already been handled in (22). In this situation we have

$$
\begin{aligned}
\mathbb{P}(\mathbf{E}: \text { GoStraight })= & (1+o(1))\left[\left(1-\mathcal{M}\left(\left[\left[\overline{x_{1} x_{0}}\right]\right]\right)\right) \sigma^{\mathcal{M}}\left[d l_{1}, x_{0} ; d l_{2}, x_{2} ; \ldots ; d l_{k}, x_{k}\right]\right. \\
& -\int_{l^{\prime} \in\left[\left[\overline{x_{1} x_{0}}\right]\right]} \sigma^{\mathcal{M}}\left[d l^{\prime}, x^{\prime \prime} ; d l_{1}, x_{0} ; d l_{2}, x_{2} ; \ldots ; d l_{k}, x_{k}\right] \\
& \left.-\sum_{l_{j} \in\left[\left[\overline{x_{1} x_{0}}\right]\right], l_{j} \neq l_{1}} \sigma^{\mathcal{M}}\left[d l_{j}, x_{j}^{\prime \prime} ; d l_{1}, x_{0} ; d l_{2}, x_{2} ; \ldots ; d l_{k}, x_{k}\right]\right],
\end{aligned}
$$

where $x^{\prime \prime}$ and $x_{j}^{\prime \prime}$ in the above integrals stand for the respective intersection points of $l^{\prime}$ and $l_{j}$ with $\partial D_{1-d s}$, by definition lying inwards from $\left\{x^{\prime}\right\}:=l^{\prime} \cap \overline{x_{0} x_{1}}$ and $\left\{x_{j}^{\prime}\right\}:=l_{j} \cap \overline{x_{0} x_{1}}$. To establish (23) note first that on (E:GoStraight) an edge $e_{1}$ in the direction of $l_{1}$ is present at $x_{0}$ at the time $1-d s$ of the dynamic construction and, in the course of the dynamic construction, during the time period $[1-d s, 1]$ the edge unfolds along $\overline{x_{0} x_{1}}$ eventually reaching $x_{1}$. Consequently, there can be no (GE:DirectionalUpdate) directional updates along $\overline{x_{0} x_{1}}$ and neither can there be (GE:Collision) collisions with other already existing edges. However, according to the (GE) dynamics such directional updates are possible along each line from $\left[\left[\overline{x_{0} x_{1}}\right]\right]$, consequently the probability that none of these possible turns occurs yields the prefactor $\exp \left(-\mathcal{M}\left(\left[\left[\overline{x_{1} x_{0}}\right]\right]\right)\right)=(1+o(1))\left[1-\mathcal{M}\left(\left[\left[\overline{x_{1} x_{0}}\right]\right]\right)\right]$ whereas the remaining factor $\sigma^{\mathcal{M}}\left[d l_{1}, x_{0} ; d l_{2}, x_{2} ; \ldots ; d l_{k}, x_{k}\right]$ in the first term in the RHS of (23) is the probability that the marker points $x_{0}, x_{2}, \ldots, x_{k}$ are covered by their corresponding 
edges as required. We claim that the resulting product

$$
(1+o(1))\left(1-\mathcal{M}\left(\left[\left[\overline{x_{1} x_{0}}\right]\right]\right)\right) \sigma^{\mathcal{M}}\left[d l_{1}, x_{0} ; d l_{2}, x_{2} ; \ldots ; d l_{k}, x_{k}\right]
$$

represents the probability that the event $\mathcal{E}\left[d l_{1}, x_{0} ; d l_{2}, x_{2} ; \ldots ; d l_{k}, x_{k}\right]$ holds and no (GE:DirectionalUpdate) turns occur along $\overline{x_{0} x_{1}}$. To see it we observe that a possible directional update of this kind would yield, during the period $[1-d s, 1]$ of the graphical construction, an outward edge $e^{\prime}$ of infinitesimal length, almost immediately hitting the boundary $\partial D_{1}$. Since for almost all time moments in the (EM) dynamics the distance between $x_{1}$ and other marker points $x_{i}, i>1$, is strictly positive, during its short evolution under the graphical construction dynamics the edge $e^{\prime}$

- is overwhelmingly unlikely to have its birth event along the infinitesimal segment $\overline{x_{0}, x_{1}}$ affected by the occurrence of $\mathcal{E}\left[d l_{1}, x_{0} ; d l_{2}, x_{2} ; \ldots ; d l_{k}, x_{k}\right]$,

- has only a negligible chance of affecting the occurrence of the considered event $\mathcal{E}\left[d l_{1}, x_{0} ; d l_{2}, x_{2} ; \ldots ; d l_{k}, x_{k}\right]$ because, in the course of the graphical construction, $e^{\prime}$ is born after $x_{0}$ and other $x_{i}$ 's contained in $D_{1-d s}$ get covered by the field and, in addition, $e^{\prime}$ is, with overwhelming probability, too short to reach neighbourhood of any of the remaining $x_{i}$ 's contained in $D_{1} \backslash D_{1-d s}$.

This nearly independence justifies taking the above product, as required. Next, we have to subtract the probability that $\mathcal{E}\left[d l_{1}, x_{0} ; d l_{2}, x_{2} ; \ldots ; d l_{k}, x_{k}\right]$ occurs and there are no directional updates (GE:DirectionalUpdate) along $\overline{x_{0} x_{1}}$ but a (GE:Collision) collision of the edge unfolding from $x_{0}$ along $l_{1}$ with another already existing edge occurs on $\overline{x_{0} x_{1}}$. There are two possible sources of such collisions

- $\overline{x_{0} x_{1}}$ meets an inward edge $e^{\prime}$ along some $l^{\prime}$ non-colinear with any of the marker lines $l_{j}$. Then the probability of the considered event is

$$
(1+o(1)) \sigma^{\mathcal{M}}\left[d l^{\prime}, x^{\prime \prime} ; d l_{1}, x_{0} ; d l_{2}, x_{2} ; \ldots ; d l_{k}, x_{k}\right]
$$

with the prefactor $(1+o(1))$ due to the requirements that there be no directional updates along $\overline{x_{0} x_{1}}$ and that the inward edge $e^{\prime}$ reaches $x^{\prime \prime}$, which are negligibly unlikely to fail over the infinitesimal time interval $[0, d s]$. This expression corresponds to the second term in (23) above.

- $\overline{x_{0} x_{1}}$ meets an inward edge $e^{\prime}$ along some marker line $l_{j}$. In analogy to the case above, here the probability of the considered event is

$$
(1+o(1)) \sigma^{\mathcal{M}}\left[d l_{j}, x_{j}^{\prime \prime} ; d l_{1}, x_{0} ; d l_{2}, x_{2} ; \ldots ; d l_{k}, x_{k}\right] .
$$

This expression corresponds to the third term in (23) above.

- If $x_{1}$ is not coupled with any other marker point $x_{j}$ then for (E:TurnOutwards) we have

$$
\mathbb{P}(\mathbf{E}: \text { TurnOutwards })=(1+o(1)) \mathcal{M}\left(d l_{1}\right) \mathcal{M}\left(\left[\left[\overline{x_{1} x_{0}}\right]\right]\right) \sigma^{\mathcal{M}}\left[d l_{2}, x_{2} ; \ldots ; d l_{k}, x_{k}\right] .
$$

Indeed, the probability of the vertex birth (GE:VertexBirth) at $x^{\prime}$ as required for (E:TurnOutwards) is $\mathcal{M}\left(d l_{1}\right) \mathcal{M}\left(d l^{\prime}\right)$, with the notation as in the above definition of the event. Integrating over $l^{\prime} \in\left[\left[\overline{x_{1} x_{0}}\right]\right]$ yields $\mathcal{M}\left(d l_{1}\right) \mathcal{M}\left(\left[\left[\overline{x_{1} x_{0}}\right]\right]\right)$. Moreover, the occurrence of such a (GE:VertexBirth) event at some dynamic construction time in $[1-d s, 1]$, as yielding an infinitesimally short outward edge $e^{\prime}$, is overwhelmingly unlikely to affect or be affected by the occurrence of $\mathcal{E}\left[d l_{2}, x_{2} ; \ldots ; d l_{k}, x_{k}\right]$ for precisely the same reasons as those justifying (24) above. This nearly independence allows us to express $\mathbb{P}\left(\mathbf{E}\right.$ :TurnOutwards) as the product of $\mathcal{M}\left(d l_{1}\right) \mathcal{M}\left(d l^{\prime}\right)$ and 
$\sigma^{\mathcal{M}}\left[d l_{2}, x_{2} ; \ldots ; d l_{k}, x_{k}\right]$ with the extra $(1+o(1))$ coming also from the requirement that there be no (GE:DirectionalUpdate) turns nor (GE:Collision) collisions along $\overline{x^{\prime} x_{1}}$.

Note that the above conclusions are valid regardless of whether some marker line $l_{j}$, $j \neq 1$, does cross $\overline{x_{0} x_{1}}$ or not. Indeed, if such $l_{j}$ crosses $\overline{x_{0} x_{1}}$ then the corresponding marker point $x_{j}$ lies in the inward direction from the intersection point for otherwise an (EM:DiscardOnCollision) event would occur in the (EM) dynamics, which we assumed not to be the case. Thus, a possible outward turn in the direction of $l_{j}, j \neq 1$, occurring along $\overline{x_{0} x_{1}}$ cannot yield an edge reaching and affecting the status of $x_{j}$ and the occurrence of $\mathcal{E}\left[d l_{2}, x_{2} ; \ldots ; d l_{k}, x_{k}\right]$.

- If $x_{1}$ is coupled with some other marker point $x_{j}, j \neq 1$, along $l_{1}=l_{j}$ then for (E:TurnOutwards) we have $\mathbb{P}(\mathbf{E}$ :TurnOutwards $)=0$ because the probability of obtaining in (GE:VertexBirth) at $x^{\prime}$ an edge $e_{1}$ exactly colinear with $l_{j}$ is zero since $\mathcal{M} \ll \mu$.

- If $x_{1}$ is not coupled with any other marker point $x_{j}$ then for (E:TurnInwards) we have

$$
\mathbb{P}(\mathbf{E}: \text { TurnInwards })=(1+o(1)) \mathcal{M}\left(d l_{1}\right) \int_{l^{\prime} \in\left[\left[\overline{x_{1} x_{0}}\right]\right]} \sigma^{\mathcal{M}}\left[d l^{\prime}, x^{\prime \prime} ; d l_{2}, x_{2} ; \ldots ; d l_{k}, x_{k}\right] .
$$

Note that if some $l_{j}, j \neq 1$, crosses $\overline{x_{0} x_{1}}$ then the integral in the RHS of (26) above includes the singular term $\mathcal{M}\left(d l_{1}\right) \sigma^{\mathcal{M}}\left[d l_{j}, x_{j}^{\prime \prime} ; d l_{2}, x_{2} ; \ldots ; d l_{j}, x_{j} ; \ldots ; d l_{k}, x_{k}\right]$ corresponding to the situation where $l^{\prime}=l_{j}$. To establish (26) observe that the probability of our edge $e_{1}$ along $l_{1}$ arising due to a (GE:DirectionalUpdate) directional update at $x^{\prime}$ on an inward edge $e^{\prime}$ along $l^{\prime}$ and of having $\mathcal{E}\left[d l_{2}, x_{2} ; \ldots ; d l_{k}, x_{k}\right]$ at the same time, is $\mathcal{M}\left(d l_{1}\right)$ (directional update probability) times $\sigma^{\mathcal{M}}\left[d l^{\prime}, x^{\prime \prime} ; d l_{2}, x_{2} ; \ldots ; d l_{k}, x_{k}\right]$ (probability of $\mathcal{E}\left[d l_{2}, x_{2} ; \ldots ; d l_{k}, x_{k}\right]$ holding and of having an edge $e^{\prime}$ along $l^{\prime}$ ending at $x^{\prime}$ and thus passing through $x^{\prime \prime}$ modulo negligible measure set) times $(1+o(1))$ to take into account the requirement that there be no further (GE:DirectionalUpdate) turns along $\overline{x^{\prime} x_{1}}$ which is satisfied with overwhelming probability. In analogy to our previous considerations for (24), also here taking products of the above probabilities, modulo $(1+o(1))$, is well justified because the directional update at $x^{\prime}$ has only a negligible chance of affecting the occurrence of $\mathcal{E}\left[d l_{2}, x_{2} ; \ldots ; d l_{k}, x_{k}\right]$. Integrating over $l^{\prime}$ yields now (26) as required.

- If $x_{1}$ is coupled with some other marker point $x_{j}, j \neq 1$, along $l_{1}=l_{j}$ then for (E:TurnInwards) we have $\mathbb{P}(\mathbf{E}$ :TurnInwards $)=0$ because the probability of obtaining in (GE:DirectionalUpdate) at $x^{\prime}$ an edge $e_{1}$ exactly colinear with $l_{j}$ is zero since $\mathcal{M} \ll \mu$.

Putting now the above observations and formulae $(21,22,23,25,26)$ together, using the definition of the edge correlations (12) and recalling that $x_{1}$ is in boundary position as assumed, we see that, with the notation introduced in the above discussion

- If the marker $\left(l_{1}, x_{1}\right)$ separates from $(\bar{l}, \bar{x})$ then

$$
\phi\left(l_{1}, x_{1} ; l_{2}, x_{2} ; \ldots ; l_{k}, x_{k}\right)=\phi\left(l_{2}, x_{2} ; \ldots ; l_{k}, x_{k}\right) .
$$

- If there is another marker point $x_{i}, i \neq 1$, say $i=2$, in boundary position and with the property that $l_{1}$ and $l_{2}$ meet in $D_{1} \backslash D_{1-d s}$ then

$$
\phi\left(l_{1}, x_{1} ; l_{2}, x_{2} ; l_{3}, x_{3} ; \ldots ; l_{k}, x_{k}\right)=(1+o(1)) \phi\left(l_{3}, x_{3} ; \ldots ; l_{k}, x_{k}\right) \text {. }
$$

- Otherwise:

- If $x_{1}$ is not coupled with any other marker point $x_{j}, j \neq 1$, 


$$
\begin{aligned}
\sigma^{\mathcal{M}}\left(d l_{1}, x_{1} ; \ldots ; d l_{k}, x_{k}\right)= & (1+o(1))\left[\left(1-\mathcal{M}\left(\left[\left[\overline{x_{1} x_{0}}\right]\right]\right)\right) \sigma^{\mathcal{M}}\left[d l_{1}, x_{0} ; \ldots ; d l_{k}, x_{k}\right]\right. \\
& +\mathcal{M}\left(d l_{1}\right) \mathcal{M}\left(\left[\left[\overline{x_{1} x_{0}}\right]\right]\right) \sigma^{\mathcal{M}}\left[d l_{2}, x_{2} ; \ldots ; d l_{k}, x_{k}\right] \\
& +\int_{l^{\prime} \in\left[\left[\overline{x_{1} x_{0}}\right]\right]}\left[\mathcal{M}\left(d l_{1}\right) \sigma^{\mathcal{M}}\left[d l^{\prime}, x^{\prime \prime} ; d l_{2}, x_{2} ; \ldots ; d l_{k}, x_{k}\right]\right. \\
& \left.-\sigma^{\mathcal{M}}\left[d l^{\prime}, x^{\prime \prime} ; d l_{1}, x_{0} ; d l_{2}, x_{2} ; \ldots ; d l_{k}, x_{k}\right]\right] \\
& \left.-\sum_{j \neq 1, l_{j} \in\left[\left[\overline{x_{0} x_{1}}\right]\right]} \sigma^{\mathcal{M}}\left[d l_{j}, x_{j}^{\prime \prime} ; d l_{1}, x_{0} ; d l_{2}, x_{2} ; \ldots ; d l_{k}, x_{k}\right]\right]
\end{aligned}
$$

whence, upon recalling the definition (13) of the edge correlation function $\phi(\cdot)$,

$$
\begin{aligned}
\phi\left(l_{1}, x_{1} ; \ldots ; l_{k}, x_{k}\right)= & (1+o(1))\left[\left(1-\mathcal{M}\left(\left[\left[\overline{x_{1} x_{0}}\right]\right]\right)\right) \phi\left(l_{1}, x_{0} ; \ldots ; l_{k}, x_{k}\right)\right. \\
& +\mathcal{M}\left(\left[\left[\overline{x_{1} x_{0}}\right]\right]\right) \phi\left(l_{2}, x_{2} ; \ldots ; l_{k}, x_{k}\right) \\
& +\int_{l^{\prime} \in\left[\left[\overline{x_{1} x_{0}}\right]\right]}\left[\phi\left(l^{\prime}, x^{\prime \prime} ; l_{2}, x_{2} ; \ldots ; l_{k}, x_{k}\right)\right. \\
& \left.-\phi\left(l^{\prime}, x^{\prime \prime} ; l_{1}, x_{0} ; l_{2}, x_{2} ; \ldots ; l_{k}, x_{k}\right)\right] \mathcal{M}\left(d l^{\prime}\right) \\
& +\sum_{j \neq 1, l_{j} \in\left[\left[\overline{x_{0} x_{1}}\right]\right]}\left[\phi\left(l_{j}, x_{j}^{\prime \prime} ; l_{2}, x_{2} ; \ldots ; l_{k}, x_{k}\right)\right. \\
& \left.\left.-\phi\left(l_{j}, x_{j}^{\prime \prime} ; l_{1}, x_{0} ; l_{2}, x_{2} ; \ldots ; l_{k}, x_{k}\right)\right]\right]
\end{aligned}
$$

where the extra positive term in the last sum comes from separate treatment of the case $l^{\prime}=l_{j}$ in (26), see the discussion directly following this display. This can be further rewritten as

$$
\begin{aligned}
\phi\left(l_{1}, x_{1} ; \ldots ; l_{k}, x_{k}\right)= & (1+o(1))\left[\left(1-2 \mathcal{M}\left(\left[\left[\overline{x_{1} x_{0}}\right]\right]\right)\right) \phi\left(l_{1}, x_{0} ; \ldots ; l_{k}, x_{k}\right)\right. \\
& +\mathcal{M}\left(\left[\left[\overline{x_{1}} x_{0}\right]\right]\right) \phi\left(l_{2}, x_{2} ; \ldots ; k_{k}, x_{k}\right) \\
& +\int_{l^{\prime} \in\left[\left[\overline{x_{1} x_{0}}\right]\right]}\left[\phi\left(l_{1}, x_{0} ; \ldots\right)+\phi\left(l^{\prime}, x^{\prime \prime} ; l_{2}, x_{2} ; \ldots\right)\right. \\
& \left.-\phi\left(l^{\prime}, x^{\prime \prime} ; l_{1}, x_{0} ; l_{2}, x_{2} ; \ldots\right)\right] \mathcal{M}\left(d l^{\prime}\right) \\
& +\sum_{\left.j \neq 1, l_{j} \in\left[\overline{x_{0} x_{1}}\right]\right]}\left[\phi\left(l_{j}, x_{j}^{\prime \prime} ; l_{2}, x_{2} ; \ldots ; l_{k}, x_{k}\right)\right. \\
& \left.\left.-\phi\left(l_{j}, x_{j}^{\prime \prime} ; l_{1}, x_{0} ; l_{2}, x_{2} ; \ldots ; l_{k}, x_{k}\right)\right]\right] .
\end{aligned}
$$

- Likewise, if $x_{1}$ is coupled with some other marker point $x_{j}, j \neq 1$, then

$$
\begin{aligned}
\phi\left(l_{1}, x_{1} ; \ldots ; l_{k}, x_{k}\right)= & (1+o(1))\left[\left(1-\mathcal{M}\left(\left[\left[\overline{x_{1} x_{0}}\right]\right]\right)\right) \phi\left(l_{1}, x_{0} ; \ldots ; l_{k}, x_{k}\right)\right. \\
& -\int_{l^{\prime} \in\left[\left[\overline{x_{1} x_{0}}\right]\right]} \phi\left(l^{\prime}, x^{\prime \prime} ; l_{1}, x_{0} ; l_{2}, x_{2} ; \ldots ; l_{k}, x_{k}\right) \mathcal{M}\left(d l^{\prime}\right) \\
& \left.-\sum_{l_{i} \in\left[\left[\overline{x_{1} x_{0}}\right]\right], l_{i} \neq l_{1}} \phi\left(l_{i}, x_{i}^{\prime \prime} ; l_{1}, x_{0} ; l_{2}, x_{2} ; \ldots ; l_{k}, x_{k}\right)\right] .
\end{aligned}
$$


To complete the above discussion we recall that in the complementary case $x_{1} \notin \partial D_{1}$ the point marker $x_{1}$ would not evolve under the (EM) dynamics.

To proceed we combine $(21,22,27,28)$ and extend these relations for all the other boundary marker points $x_{i}$ in $(\bar{l}, \bar{x})$. Recalling the evolution rules (EM) for the edge marker process, taking into account that $\Psi_{0}=\{(\bar{l}, \bar{x})\}$ and getting rid of the $(1+o(1))$ prefactors as $d s \rightarrow 0$, we finally obtain the relation

$$
\Phi_{0}=\phi(\bar{l}, \bar{x})=\mathbb{E} \sum_{\left(\bar{l}^{(p)}(d s), \bar{x}^{(p)}(d s)\right) \in \Psi_{d s}} \eta^{(p)}(d s) \phi\left(\bar{l}^{(p)}(d s), \bar{x}^{(p)}(d s)\right)=\mathbb{E} \Phi_{d s} .
$$

Observe in this context that

- the second line in (27) corresponds to (EM:Kill) rule,

- the third line there to (EM:TurnAndBranch) rule,

- and the fourth line to (EM:ForcedTurnAndBranch).

Likewise, the absence of certain terms in the coupled version (28) of (27) corresponds to annihilation of coupling-breaker configurations in (EM:UnbreakableCouplings). Clearly, the crucial relation (29) admits straightforward extensions for more general initial conditions and all time moments between 0 and 1, as discussed at the beginning of our proof. Thus, (29) implies in particular that

$$
\tilde{\Phi}_{s}:=\sum_{\left(\bar{l}^{(p)}(s), \bar{x}^{(p)}(s)\right) \in \Psi_{s}} \phi\left(\bar{l}^{(p)}(s), \bar{x}^{(p)}(s)\right), \quad s \in[0,1],
$$

where all $\eta^{(p)}$ signs are converted into pluses, is a positive submartingale. Using Lemma 3 we see it is a uniformly integrable submartingale. Therefore, noting that $\left|\Phi_{s}\right| \leq \tilde{\Phi}_{s}$, we conclude from (29) that $\left(\Phi_{s}\right)_{s \in[0,1]}$ is a martingale, which completes the proof of Lemma 4.

Completing the Proof of Theorem 2 With Lemma 4 established, we are now in a position to use the martingale representation combined with the relation (20) to complete the proof of Theorem 2 as discussed next to the statement of Lemma 4 above.

\section{Proof of Lemma 1}

Our proof is based on the so-called defective disagreement loop dynamics developed in Sect. 6.4 of [16]. Since Lemma 1 is of a purely technical rather than conceptual nature and the quite complicated defective diagreement loop dynamics finds no further applications in this paper, we decided not to present its several pages long details here, referring the reader to results of Sects. 6.4 and 7 in [16] instead. To proceed, with $(\bar{l}, \bar{x})=\left(l_{1}, x_{1}, \ldots, l_{k}, x_{k}\right)$ write

$$
\mathcal{E}\left[d l_{1}, x_{1} ; \ldots ; d l_{k}, x_{k}\right]:=\left\{\forall_{i=1}^{k} \exists_{e \in \operatorname{Edges}\left(\mathcal{A}^{\mathcal{M}}\right)} x_{i} \in e, l[e] \in d l_{i}\right\}
$$

for the correlation-defining events. We claim that for $\left(l_{i}, x_{i}\right)_{i=1}^{k} \in \Delta^{g}[k]$ in general position, upon fixing $l_{1}, x_{1} ; \ldots ; l_{k-1}, x_{k-1}$, the function

$$
\left(l_{k}, x_{k}\right) \mapsto \mathbb{P}\left(\mathcal{E}\left[d l_{k}, x_{k}\right] \mid \mathcal{E}\left[d l_{1}, x_{1} ; \ldots ; d l_{k-1}, x_{k-1}\right]\right) / \mathcal{M}\left(d l_{k}\right)
$$

is well defined, continuous and consequently locally bounded. Clearly, this will imply the statement of Lemma 1 upon inductive application for $(\bar{l}, \bar{x}) \in \Delta^{g}[k]$. The existence and local 
boundedness for $(\bar{l}, \bar{x}) \in \Delta^{s}[k]$ will then follow as well by noting that the edge correlation for a singular configuration is bounded above by the sum of all corresponding one-sided correlations and by repeating the existence and local boundedness argument given below for the case of one-sided correlations, which is a straightforward repetition omitted here to avoid unnecessary technicalities.

To establish our claim for (30) we use the defective disagreement loop dynamics of Sect. 6.4 in [16] with directional updating principle induced by an arbitrary growing family $\left(D_{t}\right)_{t \in[0,1]}$ as in (D1-4) satisfying in addition $D_{0}=\left\{x_{1}\right\}$ so that the anchor point $\mathbb{A}\left(l_{1}\right)$ coincides with $x_{1}$. In analogy to the proof of Theorem 4 in [16], see also Theorem 10 there, the conditional law of the polygonal field $\mathcal{A}^{\mathcal{M}}$ on the event $\mathcal{E}\left[d l_{1}, x_{1} ; \ldots ; d l_{k-1}, x_{k-1}\right]$ is invariant with respect to the following reversible conditional version of the defective disagreement loop dynamics, with $s$ standing for the corresponding time parameter in which the dynamics unfolds:

(Create) With intensity $\mathcal{M}\left(d l_{k}\right) d s$, on the event $\neg \mathcal{E}\left[d l_{k}, x_{k}\right]$ (that is to say if there is no field edge along $d l_{k}$ containing $x_{k}$ ) attempt to emit from $x_{k}$ a disagreement path with initial creation phase directed along $l_{k}$. Should the so generated path result in a configuration violating $\mathcal{E}\left[d l_{1}, x_{1} ; \ldots ; d l_{k-1}, x_{k-1}\right]$, discard the update, otherwise accept it.

(Annihilate) With intensity $d s$, on the event $\mathcal{E}\left[d l_{k}, x_{k}\right]$ (that is to say if there is a field edge along $d l_{k}$ containing $x_{k}$ ) attempt to emit from $x_{k}$ a disagreement path with initial annihilation phase directed along $l_{k}$. Should the so generated path result in a configuration violating $\mathcal{E}\left[d l_{1}, x_{1} ; \ldots ; d l_{k-1}, x_{k-1}\right]$, discard the update, otherwise accept it.

Note that there are no update failures arising due to cycle formation along disagreement paths in this dynamics, because the chosen directional updating rule comes from a generalised dynamic construction, see Sect. 6.4 in [16] for details. Denote now by $\pi_{\text {create }}$ the conditional probability of a successful Create update attempt during time interval $(s, s+d s)$ on the event $\neg \mathcal{E}\left[d l_{k}, x_{k}\right]$. Likewise, write $\pi_{\text {annihilate }}$ for the respective conditional probability for Annihilate update on $\mathcal{E}\left[d l_{k}, x_{k}\right]$. Clearly, by detailed balance for $\mathcal{E}\left[d l_{k}, x_{k}\right]$, we have

$$
\mathbb{P}\left(\mathcal{E}\left[d l_{k}, x_{k}\right] \mid \mathcal{E}\left[d l_{1}, x_{1} ; \ldots ; d l_{k-1}, x_{k-1}\right]\right)=(1+o(1)) \pi_{\text {create }} / \pi_{\text {annihilate }} .
$$

Note that $\pi_{\text {create }} \leq \mathcal{M}\left(d l_{k}\right) d s$, and that $\pi_{\text {annihilate }} \geq c d s$ for some $c$ uniformly positive with respect to small local displacements of $x_{k}$ and $l_{k}$ because there is some positive probability that the disagreement path initiated by annihilating the edge at $x_{k}$ does not hit any $x_{i}$, $i<k$, and thus does not lead to the violation of $\mathcal{E}\left[d l_{1}, x_{1} ; \ldots ; d l_{k-1}, x_{k-1}\right]$. Consequently, we conclude from (31) that the function in (30) is well defined and locally bounded on $\Delta^{g}[k]$. Its required continuity follows also by (31) in view of the assumed continuity of the activity measure density $m=d \mathcal{M} / d \mu$. We have thus established the desired properties of the conditional correlation in (30) which completes the proof of the lemma.

Acknowledgements The author acknowledges the support from the Polish Minister of Science and Higher Education grant N N201 385234 (2008-2010) as well as from grant of Nicolaus Copernicus University, Toruń, Poland. Special thanks are due to an anonymous referee whose remarks were helpful in improving this paper.

Open Access This article is distributed under the terms of the Creative Commons Attribution Noncommercial License which permits any noncommercial use, distribution, and reproduction in any medium, provided the original author(s) and source are credited. 


\section{References}

1. Arak, T.: On Markovian random fields with finite number of values. In: 4th USSR-Japan Symposium on Probability Theory and Mathematical Statistics, Abstracts of Communications, Tbilisi, 1982

2. Arak, T., Surgailis, D.: Markov fields with polygonal realizations. Probab. Theory Relat. Fields 80, 543579 (1989)

3. Arak, T., Surgailis, D.: Consistent polygonal fields. Probab. Theory Relat. Fields 89, 319-346 (1991)

4. Arak, T., Clifford, P., Surgailis, D.: Point-based polygonal models for random graphs. Adv. Appl. Probab. 25, 348-372 (1993)

5. Arias-Castro, E.: Interpolation of random hyperplanes. Electron. J. Probab. 12, 1052-1071 (2007)

6. Clifford, P., Nicholls, G.: A Metropolis sampler for polygonal image reconstruction. Available at: http:// www.stats.ox.ac.uk/clifford/papers/met_poly.html (1994)

7. Jensen, F.V., Nielsen, T.D.: Bayesian Networks and Decision Graphs, 2nd edn. Springer, Berlin (2007)

8. Kluszczyński, R., van Lieshout, M.N.M., Schreiber, T.: An algorithm for binary image segmentation using polygonal Markov fields. In: Roli, F., Vitulano, S. (eds.) Image Analysis and Processing, Proceedings of the 13th International Conference on Image Analysis and Processing. Lecture Notes in Comput. Sci., vol. 3615, pp. 383-390. Springer, Berlin (2005)

9. Kluszczyński, R., van Lieshout, M.N.M., Schreiber, T.: Image segmentation by polygonal Markov fields. Ann. Inst. Stat. Math. 59, 465-486 (2007)

10. van Lieshout, M.N.M., Schreiber, T.: Perfect simulation for length-interacting polygonal Markov fields in the plane. Scand. J. Stat. 34, 615-625 (2007)

11. Liggett, T.: Interacting Particle Systems. Springer, New York (1985)

12. Malyshev, V.A., Minlos, R.A.: Gibbs Random Fields: Cluster Expansions. Mathematics and Its Applications (Soviet Series). Kluwer, Dordrecht (1991)

13. Nicholls, G.K.: Spontaneous magnetization in the plane. J. Stat. Phys. 102, 1229-1251 (2001)

14. Schreiber, T.: Random dynamics and thermodynamic limits for polygonal Markov fields in the plane. Adv Appl. Probab. 37, 884-907 (2005)

15. Schreiber, T.: Dobrushin-Kotecký-Shlosman theorem for polygonal Markov fields in the plane. J. Stat. Phys. 123, 631-684 (2006)

16. Schreiber, T.: Non-homogeneous polygonal fields in the plane: graphical constructions and geometry of higher order correlations. J. Stat. Phys. 132, 669-705 (2008)

17. Schreiber, T., van Lieshout, M.N.M.: Disagreement loop and path creation/annihilation algorithms for binary planar Markov fields with applications to image segmentation. Scand. J. Stat. (2008, to appear)

18. Surgailis, D.: Thermodynamic limit of polygonal models. Acta Appl. Math. 22, 77-102 (1991) 